\title{
Selective Ablation of 3' RNA ends and Processive RTs Facilitate Direct cDNA Sequencing of Full-length Host cell and Viral Transcripts
}

Christian M. Gallardo ${ }^{1,2}$, Anh-Viet T. NguyenT ${ }^{1}$, Andrew L. Routh ${ }^{3,4}$, Bruce E. Torbett ${ }^{1,2,5,6,7 *}$.

${ }^{1}$ Department of Immunology and Microbiology, The Scripps Research Institute, La Jolla, CA, USA

${ }^{2}$ Center for Immunity and Immunotherapies, Seattle Children's Research Institute, Seattle WA, USA

${ }^{3}$ Department of Biochemistry and Molecular Biology, Sealy Center for Structural Biology, University of Texas Medical Branch, Galveston, TX, USA

${ }^{4}$ Sealy Center for Structural Biology, University of Texas Medical Branch, Galveston, TX, USA

${ }^{5}$ Institute for Stem Cell \& Regenerative Medicine, University of Washington, Seattle, WA, USA

${ }^{6}$ Department of Laboratory Medicine and Pathology, University of Washington School of Medicine, Seattle, WA, USA

${ }^{7}$ Department of Pediatrics, University of Washington School of Medicine, Seattle, WA, USA

* correspondence should be addressed to Bruce E. Torbett: bertorbet@uw.edu 


\section{$\underline{\text { Abstract }}$}

Alternative splicing (AS) is necessary for viral proliferation in host cells and a critical regulatory component of viral gene expression. Conventional RNA-seq approaches provide incomplete coverage of AS due to their short read-lengths and are susceptible to biases and artifacts introduced in prevailing library preparation methodologies. Moreover, viral splicing studies are often conducted separately from host cell transcriptome analysis, precluding an assessment of the viral manipulation of host splicing machinery. To address current limitations, we developed a quantitative full-length direct cDNA sequencing strategy to simultaneously profile viral and host cell transcripts. This nanopore-based approach couples processive reverse transcriptases with a novel one-step chemical ablation of 3' RNA ends (termed CASPR) which decreases ribosomal RNA reads and enriches for poly-adenylated coding sequences. We extensively validate our approach using synthetic reference transcripts and show CASPR doubles the breadth of coverage per transcript and increases detection of long transcripts $(>4 \mathrm{~kb})$, while being functionally equivalent to PolyA + selection for transcript quantification. We used our approach to interrogate host cell and HIV-1 transcript dynamics during viral reactivation and identified novel putative HIV-1 host factors containing exon skipping or novel intron retentions and delineated the HIV-1 transcriptional state associated with these differentially regulated host factors. 


\section{Introduction}

Alternative splicing (AS) greatly increases protein diversity encoded by the human genome, and has been estimated to occur in up to $95 \%$ of genes with multiexonic transcripts (1). This process is tightly regulated by cisand trans- acting elements, chromatin accessibility, and other signaling pathways (2). Alternative splicing has been shown to be a driver of human proteome diversity $(3,4)$ and a critical regulatory component in the tissuespecific expression of human transcriptomes(5). Recently, increasing use of massively parallel RNA-Seq pipelines have allowed population-scale transcriptome studies which have revealed naturally occurring variants that modulate AS and influence disease susceptibility(6).

Viral infections commonly alter host cell splicing landscape, as shown by genes that appear differentiallyspliced upon viral infection in transcriptomic studies, or splicing-related genes that appear differentially enriched or phosphorylated in proteomic studies (7). In cells infected with HIV-1 (HIV), alternatively-spliced host cell transcripts have been shown to promote a permissive environment for viral activation and proliferation via induction of alternative transcription start/end sites (8) and via functional enrichment of HIV replication related pathways (9). Similarly, proteomic studies have shown induction of signaling pathways involved in mRNA splicing in T-lymphocytes upon HIV entry (10), with phosphorylation of canonical splice factors being the apparent regulatory mechanism. Additionally, splicing-related host factors have been reported which bind HIV accessory proteins and act as trans-regulatory elements including the binding of U2AF65 and SPF45 by Rev (11) and SR proteins by $\mathrm{Vpr}$ (12), as well as the interactions between POLR2A and Tat (13).

Alternative splicing is also a critical regulatory mechanism of viral gene expression (14). In HIV, a single unspliced 9.2kb RNA serves as both the genome and mRNA for both Gag and Gag-Pol polyproteins, while alternatively-spliced mRNA variants code for the 7 remaining gene products by dynamically and specifically interacting with regulatory elements, thereby generating over 50 physiologically relevant transcripts that can be grouped in 'partially spliced' (4kb) and 'completely spliced' (1.8kb) groups (15-18). The underlying mechanism in AS regulation of HIV transcripts is the placement of the open-reading frames of each gene in close proximity to the single transcription start site region at the 5' end of HIV-RNA, thus optimizing the coding potential of HIVgenes by translating different proteins from a common mRNA. The completely spliced $1.8 \mathrm{~kb}$ class is particularly important during the early infection phase, and it includes Tat and Rev transcripts which respectively aid in transcription and export of partially spliced transcripts from the nucleus. An eventual shift in splicing dynamics, partially attributed to Rev, results in increased production of partially spliced and unspliced mRNAs (11). Thus, carefully orchestrated splicing dynamics are critical for regulating the dynamics of HIV gene expression and any resulting interactions with host factors.

Conventional RNA-seq approaches, while robust and reproducible, are limited by their read-length in providing full coverage of AS events (such as alternate donor/acceptor sites, exon skipping, alternate exon usage, and intron retention). Moreover, prevailing library preparation techniques introduce biases/artifacts due to PCR amplification bias, artefactual recombination, fragmentation, or targeted enrichment methods for coding sequences (CDS) (19). The read-length limitation in short-read RNA-seq, coupled with the biases and artifacts introduced in prevailing library preparation methodologies can prevent a quantitative assessment of full exon connectivity in a quantitative manner, resulting in loss of information on transcript isoform diversity, including splice variants (20). The limitations of current RNA-seq approaches are particularly exacerbated when assessing transcript expression in polycistronic HIV RNA where all transcripts are flanked by identical 5' and 3' end exons (only varying in their internal splicing sites) and vary greatly in overall transcript length. Previous attempts to address these constraints have used primer sets for each transcript class or gene product, relied on molecular barcoding, or emulsion PCR to ameliorate PCR skewing or sampling biases $(18,21)$. However, use of different primer sets prevents the quantitative comparison between transcripts and does not provide full exon coverage, while molecular barcoding approaches were used with short-read NGS approaches. Previous HIV splicing studies were not implemented within the context of a host cell transcriptome analysis, precluding a direct assessment of the viral manipulation of host splicing machinery or further insights into virus-host interaction dynamics (22). Since the regulation of HIV gene expression depends on the ability of the virus to co-opt host cell splicing machinery, understanding host cell transcriptional state and its resulting HIV mRNA-splicing signature would identify novel molecular signatures of HIV infection and provide opportunities for drug/probe development based on novel viral/host factor interactions. 
To address current RNA-seq limitations, we developed and validated a quantitative full-length RNA-seq strategy for the simultaneous profiling of poly-adenylated host and viral transcripts from unamplified cDNA. The nanopore sequencing based approach is supported by use of processive reverse transcriptases (RTs), such as MarathonRT $(23,24)$, and oligo-d(T) priming, coupled with a one-step chemical ablation of 3' RNA ends (named CASPR) which decreases ribosomal RNA reads and enriches for poly-adenylated transcripts. We validate RT conditions to provide for full-length transcripts for sequencing and CDS enrichment strategies using synthetic reference transcripts and show that while CASPR is functionally equivalent to PolyA+ selection for transcript quantification purposes, it provides critical advantages in doubling the breadth of coverage per transcript and significantly increasing the efficiency of capture of long transcripts $>4 \mathrm{~kb}$ in size. This improved practical throughput and likelihood of capturing full-exon connectivity. We then demonstrate the utility of our pipeline by interrogating host cell and HIV transcript dynamics in reactivated J-Lat 10.6 cells, a widely-used cell-line model of HIV reactivation $(25,26)$. We identify putative host factor correlates of HIV transcriptional reactivation that contain exon skipping events (PSAT1) or novel intron retentions (PSD4) and delineate the HIV transcriptional state associated with these differentially regulated host factors. We anticipate this pipeline will allow greater insights into host cell-pathogen transcript dynamics involved in viral infection and activation. 


\section{$\underline{\text { Materials and Methods }}$}

\section{Cell Culture}

J-Lat 10.6 cells, a Jurkat-derived cell line that is latently infected with HIV(25), were obtained from the NIH AIDS Reagent Program (clone \#10.6, Dr. Eric Verdin). The J-Lat 10.6 clone contains a single R7/ $\Delta$ Env strain integrated into the SEC16A locus, and EGFP inserted into the nef ORF. For control experiments, the Jurkat E61 clone was obtained from the NIH AIDS Reagent Program (cat \#177, from Dr. Arthur Weiss(27)). J-Lat 10.6 cells were activated with 10ng/mL TNF-alpha (PeproTech 300-01A) for 24 hours which induces latency reversal of integrated provirus, resulting in positive GFP expression and p24 production which are respectively detected via flow cytometry and p24 ELISA. Cell lines were maintained in RPMI 1640 (Life Tech) supplemented with $10 \%$ FBS (Hyclone) and $1 \%$ Pen/Strep at $37^{\circ} \mathrm{C}$ and $5 \% \mathrm{CO}_{2}$.

\section{Total RNA isolation}

Total RNA was isolated from cell pellets $\left(<1 \times 10^{7}\right.$ cells) using the RNeasy Mini kit (QIAGEN, cat. 74134). Cells were lysed with RLT buffer (with no ß-ME) and processed according to manufacturer's instructions, and eluted in 25-50 $\mu \mathrm{L}$ nuclease free water. Total RNA sample quality was assessed via Agilent Bioanalyzer using the RNA 6000 Nano kit, resulting in RNA Integrity Scores (RIN) of 10, for all samples.

\section{Synthetic RNA Reference Standards}

Spike-In RNA Variants (SIRVs) that include Iso Mix E0, ERCC, and Long SIRV modules were purchased from Lexogen (SIRV-Set 4, 141.03). SIRVs were resuspended in $10 \mu \mathrm{L}$ Nuclease-free water to a concentration of 5.35 ng/ $\mu \mathrm{L}$. Resuspended SIRVs were then admixed into total RNA preparations prior to PolyA+ selection, CASPR treatment, or Reverse Transcription, to a concentration of $0.13 \mathrm{ng}$ of SIRVs per $\mu \mathrm{g}$ of total RNA sample.

\section{Chemical Ablation of 3' RNA ends}

Sodium Periodate $\left(\mathrm{NaIO}_{4}\right)$ was purchased from Millipore Sigma (311448-5G). A 2X Buffered Periodate Solution (BP) is prepared fresh each time by measuring $\mathrm{NaIO}_{4}$ powder and resuspending to a concentration of $4 \mathrm{mg} / \mathrm{mL}$ in aqueous solution of $200 \mathrm{mM}$ Sodium Acetate pH 5.5 (Invitrogen, AM9740). Input RNA (up to $5 \mu \mathrm{g}$ ) is mixed with an equal volume of $2 \mathrm{X} \mathrm{BP}$ and incubated at room temperature in the dark for 30 mins. Following treatment, RNA is cleaned with RNA RNA Clean \& Concentrator (Zymo Research, R1013) according to the manufacturer's instructions, and eluted in Nuclease Free Water.

\section{PolyA selection}

Poly-adenylated transcripts were enriched from total RNA using the NEBNext Poly(A) mRNA Magnetic Isolation Module (E7490S), according to the manufacturer's instructions.

\section{Reverse Transcription and Second Strand Synthesis}

Reverse transcription is carried out with SuperScript IV Reverse Transcriptase (ThermoFisher, 18090010) or MarathonRT (Kerafast, EYU007) . Reactions are carried out in a $20 \mu \mathrm{L}$ volume with the following components and final concentrations: $1 X$ Reaction Buffer, dNTPs $(0.5 \mathrm{mM})$, RNAseOUT $(2 \mathrm{U} / \mu \mathrm{L})$, Oligo-d(T) primer $(1 \mu \mathrm{M})$ or $4609 \mathrm{bp}$ gene specific primer $(0.1 \mu \mathrm{M}), 5 \mathrm{mM}$ DTT (for SuperScript IV only), RNA input $(<5 \mu \mathrm{g}$ ), and MarathonRT $(0.5 \mu \mathrm{M}$ or $20 \mathrm{U})$ or SuperScript IV RT (200 U). Primers are initially annealed to template RNA in the presence of dNTPs, by heating to $65^{\circ} \mathrm{C}$ for $5 \mathrm{~min}$, followed by snap cooling to $4{ }^{\circ} \mathrm{C}$ for 2 mins. After snap cooling, the rest of the components are added, followed by reverse transcription for 1.5 hours at $42^{\circ} \mathrm{C}$ for MarathonRT and $50^{\circ} \mathrm{C}$ for SSIV. Reactions are stopped by heat inactivation at $85^{\circ} \mathrm{C}$ for 5 mins. Second strand synthesis is carried out using a modified Gubler and Hoffman procedure (28) adapted from Invitrogen's A48570 kit, in a single pot format involving direct addition of second strand buffer, dNTPs, E.coli DNA Pol I, RNAse H, and E.coli DNA Ligase to the heat inactivated first strand reaction. Second-strand synthesis is carried out at $16^{\circ} \mathrm{C}$ for 2 hours, followed by DNA Clean with the Monarch kit for downstream processing. Verification of yield and quality of cDNA is determined via NanoDrop spectrometry, and by running on an $0.8 \%$ E-Gel NGS and imaged using Azure c600 (Azure biosystems) 


\section{Nanopore Sequencing}

All samples were barcoded with Native Barcoding kit (EXP-NBD104) prior to Nanopore library preparation using the Ligation Sequencing Kit (SQK-LSK109). All samples sequenced with MinION R9.4.1 flowcells, basecalled with Guppy basecaller 3.4.5, and demultiplexed with Guppy barcoder.

\section{Reference Sequences}

A custom ribosomal RNA reference file was created by concatenating the fasta sequences for 28S (Gene ID: 100008589), 5.8S (Gene ID: 100008587), 5S (Gene ID: 100169751) and 18S (Gene ID: 100008588) ribosomal RNA sequences. IncRNA transcripts in fasta format were downloaded from Gencode release 31 (GRCh38.p12). For Human Reference alignment the UCSC analysis set of Dec. 2013 human genome (GCA_000001405.15) without the alt-scaffolds was used along with its associated gtf annotation file when appropriate. A custom reference sequence for R7 viral strain present in J-Lat cells was generated by extracting mapped reads from previous HIV alignments, size filtering, assembling with Unicycler (https://github.com/rrwick/Unicycler), polished with Medaka, and manually inspected with SnapGene against HXB2 originating background sequence to rule out structural variants.

\section{Determination of uniquely mapped reads}

Reads were mapped to rRNA reference using minimap2 with map-ont preset. Unmapped reads were extracted from the sam output using samtools view followed by conversion to fastq using samtools bam $2 f q(29)$. Fastq file containing unmapped rRNA reads were mapped to lncRNA reference with minimap2 using splice preset, followed by extraction of unmapped reads and conversion to fastq as before. Unmapped lncRNA reads were remapped to human reference with minimap2 using splice preset. Uniquely mapped reads were counted for each resulting sam file using samtools view with -F260 flag to only count primary alignments and the $-c$ option to output number of reads.

\section{Gene Body Coverage, Splice Junction Number, Read Distribution}

For Gene Body Coverage calculation(30), reads were mapped directly to hg38 analysis set reference using minimap2 with splice preset and --secondary=no flag, with mapped reads converted to bam format, sorted and indexed using samtools. Gene Body Coverage is calculated with the geneBody_coverage.py script that is part of the RSeQC package (v3.0.1) using sorted and indexed bam files and the UCSC RefSeq (refGene) annotations in bed format. Splice junction quantification and saturation was calculated using the junction_saturation.py script, also within RSeQC package, and with identical inputs as before. For Intragenic and Intergenic read distributions, reads were mapped and processed as before using the gencode v31 human reference (GRCh38.p12). The comprehensive genome annotation gtf file was collapsed using GTEx collapse annotation script. Read distributions were computed from mapped reads and collapsed annotations using RNA-SeQC (v2.3.4) with the following options --unpaired --coverage -base-mismatch=180 --mapping-quality 0 --detection-threshold=0 -legacy.

\section{Statistical Analysis}

Where indicated, $\mathrm{t}$-tests were run between CASPR and PolyA-selected samples within RT enzyme group (either MRT or SSIV). Analyses performed within GraphPad Prism 8, assuming all rows are sampled from populations with same scatter (SD). Statistical significance determined using the Holm-Sidak method, with alpha $=0.05$. Statistical significance denoted as following: $\mathrm{p}<0.05(*), \mathrm{p}<0.01(* *), \mathrm{p}<0.001(* * *), \mathrm{p}<0.0001(* * * *)$

\section{HIV isoform analysis}

Reads were mapped to R7 reference sequence with minimap2 using splice preset, followed by filtering using F260 flag in samtools view and sorting. Resulting sorted bam file is used as input for Pinfish pipeline (https://github.com/nanoporetech/pinfish). Briefly bam files were used as input for spliced_bam2gff command using the $-M$ option. The resulting gff file is clustered into isoform bins using cluster_gff command using the following options - $c 3-p$ 0. Isoforms clusters are then polished using polish_clusters command with $-c$ option. Polished clusters in fasta format are remapped to reference using minimap2 and processed using same settings as before. Polished clusters are visualized at this stage using IGV 2.7.2, and coverage maps for clustered isoforms 
are obtained with the samtools depth command with the $-a-d 0$ options. The spliced_bam2gff command is then run with identical options as before and resulting polished clusters that are then collapsed with the collapse partials command with the $-M-U$ options. Resulting GFF files are then manually parsed to retain those isoforms that contain at least one full CDS.

\section{Host cell transcript isoform analysis}

Analysis of host cell isoforms was performed using the FLAIR pipeline (31) v1.4. Reads are mapped to UCSC hg38 reference using flair align module using option - $p$, followed by splice junction correction with the flair correct module. Isoforms are collapsed using the flair collapse module with --stringent --trust_ends options to ensure $80 \%$ coverage per isoform cluster. Transcript lengths can be calculated with flair collapse outputs, by indexing the transcripts.fa file for each sample with samtools faidx and extracting the second column containing length of each sequence. The isoforms are then quantified with the flair quantify module using --tpm --trust_ends options. Outputs of this module were used to compute gene expression TPM correlation between samples and replicates. The flair diffexp module is finally used to generate differential gene/isoform expression analysis with default settings. Finally, the flair diffsplice module is used to determinate high confidence alternative splicing events from the isoforms processed with previous modules. Differential gene, isoform or splicing outputs are filtered for max p-value of 0.1 , those hits that remain are subject to additional FDR analysis with those with $p$ adj $<0.1$ being highly significant. Transcript discovery sensitivity and specificity was calculated using gffcompare v0.11.5(32) using gtf files outputs from flair collapse module and the UCSC hg38 genome annotation in gtf format with the following command options $-T-M-r$. 


\section{$\underline{\text { Results }}$}

\section{Improvement of the specificity and yield of high performing reverse transcriptases for producing full- length transcripts for direct cDNA sequencing}

Obtaining a readout of alternative splicing of host and viral transcripts involves end-to-end sequencing reads which provides for full-exon connectivity. To achieve this, processive reverse transcriptases are required, along with an enrichment scheme to select for protein coding sequences (CDS) from total RNA isolates. For direct cDNA sequencing, an additional requirement is to maximize the yield of cDNA so as to dispense with the need for PCR amplification of transcripts. Taking into account these requirements, we first evaluated the high performing reverse transcriptases MarathonRT (MRT), a eubacterial group II intron that has been shown to efficiently copy structured long RNAs $(23,24)$, and SuperScript IV (SSIV), which has been considered a "commercial gold standard" (33,34), for their yield of protein coding transcripts from Nalm6 total RNA, a human leukemic B cell line.

Gel electrophoresis of double-stranded cDNA obtained via SSIV and MRT showed prominent bands of similar size to ribosomal RNA when Nalm6 total RNA is directly reverse transcribed with Oligo-d(T) priming without any CDS enrichment strategy (i.e. Control) (Figure 1A). The presence of putative rRNA bands when using total RNA was unsurprising given these structural RNAs are a major RNA cellular component (enriched up to $90 \%$ in total RNA) and source of interference in RNA-Seq workflows $(35,36)$. However, ribosomal RNAs are not polyadenylated, which raises the question on the source of this spurious priming. We hypothesized these primer-independent products were the result of the RNAs themselves priming the RT initiation complexes, and that blocking 3'-OH ends of RNA inputs prior to reverse transcription could be beneficial in increasing the specificity of RT priming. For this purpose, we developed an approach dubbed Chemical Ablation of Spuriously Priming RNAs (CASPR) based on the oxidation of vicinal 2'- and 3'-OH diols of RNA, which results in the ablation of 3'-OH ends only in RNA, preventing their priming during RT and favoring RT initiation from the intended exogenous Oligo-d(T) DNA primer. Pre-treatment of input RNA with CASPR visibly improved RT specificity in both SSIV and MRT, resulting in a smear reminiscent of PolyA + selection (PolyA + ) (Figure 1A), albeit with greater mass yield compared to this established methodology (Figure 1B). The increases in specificity of Oligo-d(T) priming elicited by CASPR treatment were particularly evident in MRT samples, where CASPR treated lanes do not show any discernable rRNA bands, compared to residual rRNA bands present with SSIV. CASPR treatment also resulted in 5- and 10-fold improvements in cDNA yield compared to PolyA+ for SSIV and MRT respectively ( $\mathrm{p}<0.01$ and $\mathrm{p}<0.001)$, with the CASPR MRT combination resulting in $50 \%$ greater cDNA yield compared to CASPR SSIV ( $<<0.05$ ). This increase in RT specificity was consistent when using total RNA from other human cell lines, and when using gene-specific priming modalities with in vitro transcribed HIV RNA (Supplementary Figure 1), suggesting spurious priming from RNA inputs is prevalent.

To validate that CASPR treatment was reducing rRNA, cDNA samples were sequenced with Oxford Nanopore Technologies (ONT) MinION to determine the effect of CASPR treatment at the read mapping level (Figure 1C). As expected, the most prominent effect of CASPR treatment is the reduction of reads mapping to rRNA reference from $84 \%$ to $24 \%$ in SSIV and from $75 \%$ to $12 \%$ for MRT respectively ( $\mathrm{p}<0.0001$ for both). This reduction in rRNA-mapped reads in CASPR-treated samples was associated with a proportional increase in percent of reads mapping to the human genome (hg38) reference, from 10\% to $55 \%$ in SSIV and from $18 \%$ to $66 \%$ in MRT ( $<0.0001$ for both), which compares favorably with hg38 enrichment levels in PolyA+ samples (75-80\%). Compared to PolyA+, reads mapped to lncRNA mapping fractions were mostly nominal after CASPR treatment in both SSIV and MRT samples. Despite substantial CASPR-elicited increases in Oligo-d(T) priming specificity, the reductions in rRNA were not fully penetrant compared to PolyA+, which routinely reduced rRNA reads to $\sim 1 \%$ irrespective of RT used. However, the read mapping fractions also show that each RT is not equally susceptible to rRNA interference, with MRT showing 2-fold lower rRNA fractions and 20\% higher hg38 fractions after CASPR treatment compared to SSIV, suggesting MRT is more amenable to the priming specificity improvements elicited by ablation of 3' RNA ends when using total RNA inputs. Given improvements observed in mapped read distributions elicited by CASPR, we next evaluated its effect on the distribution of intergenic and intragenic reads (Figure 1D). As expected, the most notable effect of CASPR and PolyA+ was a dramatic reduction in intergenic reads, with an associated increase in proportion of reads mapping to exonic and intronic 

available under aCC-BY-NC-ND 4.0 International license.

regions ( $\mathrm{p}<0.0001$ for all comparisons). Interestingly, both CASPR and PolyA + slightly reduced read mappings to UTR regions in both RTs despite the associated increases in exonic reads that were observed for either treatment. All of this points to largely equivalent effects of CASPR and PolyA+ in increasing proportion of reads mapping to the intragenic features that delineate exon connectivity.

In addition to mapping statistics, the coverage along the length of protein coding transcripts is critical to reveal full exon connectivity. For this purpose, hg38 mapped reads were cross-referenced with the RefSeq genome annotation file to delineate the coverage along the 5' to 3' axis of each expressed transcript, an approach known as gene body coverage (30). The gene body coverage when using total RNA without CDS enrichment shows inconsistent coverage, with the Control SSIV samples having clear 5' and 3' end biases (and associated low coverage in middle region of gene body), and Control MRT showing consistent 3' end bias (Figure 1E). Conversely, PolyA+ samples show even coverage with normalized coverage values consistently not dipping below 0.8 across 70\% of gene body for both SSIV and MRT (Figure 1F) and no appreciable 5' or 3' end biases. These data suggest that the reduction in ribosomal RNA reads does not only increase the number of reads mapping to protein-coding transcripts, but also increases their evenness of coverage across the length of the transcript. Interestingly, MRT samples that are CASPR treated show a gene body coverage distribution similar to that observed in PolyA + samples, also consistently above 0.8 normalized coverage across majority of transcript body (Figure 1F). However, this same effect is not observed with CASPR-treated SSIV samples, with 0.5 median coverage compared to the $>0.7$ coverage values observed for all other treatment and RT combinations (Figure 1F). Overall, this data underscores the importance of CDS enrichment strategies and processive RTs in obtaining full-exon connectivity, while highlighting potential benefits of CASPR as an alternative to PolyA+ selection to substantially increase RT yield and priming specificity when using total RNA inputs.

\section{Analytical Performance Validation of CDS-enrichment strategies and RT conditions using synthetic RNA reference standards}

Initial optimization of RT conditions using processive enzymes and a novel CDS enrichment strategy suggests that the combination of MarathonRT with CASPR is well suited for direct cDNA sequencing using ONT. However, despite compelling data showing CASPR as a higher-yield analogue of PolyA+ selection, and the coverage improvements elicited with MarathonRT, neither of these interventions has been formally validated with reference standards. Synthetic RNA reference standards, which include ERCCs, SIRVs, and Sequins, have recently emerged for validating full RNA-Seq workflows (37), and contain synthetic poly-adenylated monoand/or multi-exonic transcripts of varied characteristics and in known concentration ranges. Given the synthetic nature of these transcripts, resulting reads obtained via sequencing can be cross-referenced with ground-truth annotations to evaluate quantitative features of our workflow, the sensitivity and breadth of transcript capture, length biases due to RT processivity constraints, and other performance variables. We used a Spike In RNA Variants (SIRV-Set 4) mix that was spiked into Nalm6 total RNA isolations prior to any enrichment interventions or RT with the goal of validating analytical performance of MarathonRT and CASPR against established gold standards in the field.

Consistent with previous findings, direct cDNA sequencing of SIRV-spiked Nalm6 showed that CDS enrichment strategies are critical for enrichment of poly-adenylated synthetic transcripts (Figure 2A). Specifically, CASPR treatment of total RNA prior to RT increased SIRV mapping by 5-fold in SSIV and 2.5-fold in MRT $(p<0.001$ and $p<0.01$ respectively). Moreover, the enrichment of SIRV reads with CASPR was comparable with that of PolyA+, with differences between enrichment strategies for each RT not statistically significant for SSIV and modest for MRT $(\mathrm{p}<0.05)$. Given the lack of meaningful SIRV mapping fractions without CDS enrichment, CASPR and PolyA+ samples were sequenced deeper to allow for more sensitive analysis. One such analysis involves the quantification of ERCC controls within the SIRV mix, which are present in known concentrations spanning 6-logs. Cross-referencing of measured expression of ERCC transcripts with known input amounts, showed that cDNA measurements are quantitative, with R2 values averaging 0.9 for all CDS enrichment strategies and RT combinations (Figure 2B). This robustness in cDNA quantitation translates to actual measurements of human transcript abundance with all TPM correlations strongly trending in a linear manner irrespective of RT or CDS enrichment strategy tested (Figure 2C). ERCC data and hg38 gene expression correlations are strongly suggestive of CASPR treatment being functionally equivalent to PolyA+ selection with regards to ability to 
accurately quantify cDNA levels despite residual rRNA and marginally lower hg38 mapping fractions (Figure 1C). However, this does not provide clarity on the extent of coverage of these transcripts, a critical variable for full-length sequencing.

Isoform-level analysis can add an additional layer on the breadth of transcript coverage elicited by different RTs and CDS enrichment strategies. Isoform collapse and quantification of SIRV transcripts using FLAIR (31), followed by cross-referencing to known SIRVome annotation files shows that transcript capture sensitivities are largely equivalent between CASPR and PolyA+; however, CASPR provides distinct improvements in the transcript discovery sensitivity at the Base and Locus Level (Figure 2D). Specifically, CASPR treatment shows 2-fold higher transcript discovery sensitivity at the Base level compared to PolyA with both SSIV and MRT $(p<0.001$ and $p<0.0001$ respectively) and 40-60\% higher at the Locus level ( $p<0.05$ for SSIV, $p<0.0001$ for MRT). This suggest that even though CASPR and PolyA result in equivalent number of read counts per transcript, CASPR provides significantly higher coverage per captured transcript, resulting in increased practical throughput and higher likelihood of capturing full-exon connectivity. Finally, Long SIRVs ranging from 4-12 kb were quantified after sequencing for all RTs and CDS enrichment conditions to evaluate the propensity of each treatment combination to result in size biases related to the inherent processivity constrains of RTs for RNA inputs greater than $5 \mathrm{~kb}$ in length, which was previously reported by us (24). Compared to PolyA+, CASPR trended toward increased sensitivity for capture of long synthetic transcripts greater than $5 \mathrm{~kb}$ in size for all size classes (Figure 2E). Of particular note is the statistically significant increase in sensitivity of capture of $8 \mathrm{~kb}$ transcripts elicited by CASPR treatment, resulting in 6-fold increases in capture for both SSIV and MRT ( $p<0.01$ and $p<0.0001$ respectively), and with MRT resulting in 2-fold higher sensitivity for this transcript size class as compared to SSIV $(\mathrm{p}<0.0001)$. This increase in sensitivity of capture in CASPR treated samples also translated to increased breadth of coverage for all transcript classes, with MRT in combination with CASPR showing more even coverage across all Long SIRV transcript size classes, compared to limited coverage obtained with PolyA+ for both RTs (Figure 2F). Overall, this data validates CASPR is functionally equivalent to PolyA+ selection while providing distinct advantages such as greater transcript coverage sensitivity and greater capacity to capture long transcripts. In addition, this data confirms that MarathonRT, in combination with CASPR, has superior sensitivity and breadth of coverage than SSIV for capturing long polyadenylated transcripts from complex mixtures of host cell mRNAs.

\section{Evaluation of RT and CDS enrichment strategies in the J-Lat $10.6 \mathrm{~T}$ cell line undergoing active HIV transcription}

To determine whether our direct cDNA sequencing workflow can effectively capture HIV RNAs within a swarm of host cell transcripts, we evaluated both RTs and CDS enrichment conditions using the J-Lat 10.6 lymphocytic CD4 T cell line(25). This established and well-characterized Jurkat cell line has a single integrated provirus that contains all canonical splice sites and can be robustly induced to produce viral RNAs with TNFalpha or other suitable HIV reactivation agents (26). Moreover, activation results in production of physiological levels of viral RNA, while also being representative of host transcriptional regulation dynamics of active infection(25). Thus, the J-Lat 10.6 cell line provides a stringent test case for evaluating efficiency of viral isoform capture within dynamically changing host cell transcripts without relying on PCR amplification to enrich for rare transcript variants, while allowing us to examine the effects of HIV reactivation on host cell transcript regulation.

J-Lat 10.6 cells were induced with $10 \mathrm{ng} / \mathrm{mL}$ TNF-alpha for 24 hours, followed by assessment of p24 induction and EGFP expression, with all induction values normative to previous publications (Supplementary Figure 2). Both SSIV and MRT were tested for their performance with CASPR or PolyA selection, with all replicates and samples run in parallel. As consistent with previous data, host cell gene expression TPM values show concordance between CASPR treatment and PolyA+ selection when using either SSIV or MRT (Figure 3A) and was reproducible across replicates (Supplementary Figure 3). Normalized gene body coverage values are consistent with those found in Nalm6 datasets, with CASPR MRT samples approaching the evenness observed in PolyA+ selected samples, and with SSIV showing measurable 5' end bias as consistent with previous data (Figure 3B). Compared to PolyA+, CASPR increases the fraction of long transcripts $>4000$ bp by 2.5 -fold and 6-fold in SSIV and MRT respectively ( $\mathrm{p}<0.05$ for both), in a manner that is consistent with previously observed enrichments of Long SIRVs. The ability to capture longer transcripts positions CASPR well for the capture of 
HIV transcripts which are intrinsically difficult to reverse transcribe given high RNA structure (38) and their relatively long length (2-4 kb for spliced viral transcripts) compared to host cell coding transcripts $(\sim 1 \mathrm{~kb}$ average size).

With regards to the capture efficiency of HIV transcripts, our pipeline was able to capture thousands of HIV reads despite constituting less than 1\% of total dataset (Supplementary Figure 4). To compare the performance of RTs and CDS enrichment strategies in coverage evenness, reads were mapped to the HIV reference and normalized across length of the genome, with a normalized coverage of 1 indicating even sampling (Figure 3D). CASPR and SSIV shows more consistent coverage across length of genome, with relative coverage being close 1 for most of the genome tract length relevant to multiexonic transcripts $(5,000-10,000 \mathrm{bp})$. SSIV PolyA trails closely behind, but shows reduced coverage in regions associated with Vif and Vpr transcripts (5000$6000 \mathrm{bp}$ ), and overall lower coverage for regions coding for Gag and Gag-Pol. Compared to SSIV, MRT shows 3' end bias and with coverage dropping between 7500-8300 bp. Of particular note, all samples show sharp increases in coverage at $\sim 2700$ and $\sim 200$ bp which are inconsistent with any splice junctions. However, the presence of long poly-adenylated stretches in these two regions are suggestive of mispriming of Oligo-d(T) being responsible for these artefactual increases in coverage.

To evaluate HIV isoform diversity in all treatments, HIV-mapped reads were grouped by exon boundaries into isoform clusters and collapsed into high confidence multiexonic transcript models. This analysis pipeline worked robustly and identified splice sites that were consistent with those previously observed with long-read sequencing approaches (Supplementary Table 1). Multiexonic transcripts identified by Pinfish were then parsed to determine likely expressed gene based on which undisrupted open reading frame (ORF) is closest to the 5' end (Figure 3E). As consistent with normalized coverage data, MRT with PolyA+ selection did not capture overall HIV isoform diversity, with fully-spliced species being favored. MRT with CASPR treatment performs nominally better than PolyA selection in increasing the isoform diversity of fully spliced transcripts; however, this treatment combination does not capture any partially unspliced transcripts coding for Env, Vpr and Vif. SSIV in combination with CASPR shows overall highest HIV isoform diversity, resulting in an assortment of fully-spliced transcripts and 2-3 fold higher capture of partially spliced species compared to PolyA + . The detectable differences in viral isoform diversity captured with MRT and SSIV highlight the need to evaluate each RT enzyme independently of their performance in the capture of host cell transcripts and adopt strategies that take advantage of each RT's unique characteristics and strengths. For this purpose, an optimized approach to increase the likelihood of capturing both host and viral samples would rely on the interrogation of CASPR-treated total RNA using both SSIV and MRT, followed by the simultaneous sequencing of resulting cDNA.

\section{Differential expression analysis using optimized RT and CDS enrichment conditions identifies alternatively-spliced host factors of HIV assembly and defines their associated HIV splicing signature.}

Having critically evaluated the role of CASPR in increasing transcript capture efficiency and coverage metrics, and the identified strengths of SSIV and MRT for capture of respective viral and host transcripts, we set out to do a larger scale survey of viral reactivation dynamics within host cells in the J-Lat 10.6 cell line. The goal is the simultaneous identification of differentially regulated transcripts within host cells and their HIV isoform correlates. Taking into account our previous findings regarding the unique suitability for SSIV and MRT in the efficient capture of respective viral and host transcripts, total RNA will be treated with CASPR and then split evenly to be reverse transcribed with SSIV and MRT, with resulting cDNA being used for sequencing. Since TNF-alpha induction is likely to cause global perturbations in host cell gene expression, the effect of TNF-alpha in J-Lat 10.6 case group will be compared with the differentially regulated transcripts elicited by TNF-alpha treatment in a control group of parental Jurkat cells lacking an integrated provirus. Those transcripts found to be differentially regulated by TNF-alpha in Jurkat control group, will be 'subtracted' out from those differentially regulated in J-Lat 10.6 case group, which we expect will provide greater clarity on the host-cell transcripts that are uniquely up/down regulated by active HIV transcription, and not by the HIV reactivation agent itself.

An initial pilot run, showed suitability of the approach in using both MRT and SSIV to maximize respective host cell and viral transcript capture efficiencies and coverage breadth during sequencing. Specifically, MRT showed 4-fold lower capture of artefactual rRNA-related hits in pilot differential isoform expression (DIE) analysis as compared with SSIV, with the latter showing $\sim 40 \%$ of DIE hits can be traced to rRNA loci 
(Supplementary Figure 5). Given these initial results confirming suitability of our split MRT/SSIV approach, we proceeded to sequence additional biological replicates (up to a total of 5) in the presence or absence of TNFalpha for both J-Lat (Case) and Jurkat (Control) groups (Supplementary Table 2). Differential gene expression (DGE) analysis upon TNF-alpha induction in both case and control groups with ( $p$-values $<0.1$ ), revealed 244 and 139 genes passed this filtering criteria in J-Lat case and Jurkat control groups respectively (Supplementary Figure 6). Of those genes passing p-value filtering criteria, 20 genes were found to be modulated by TNF-alpha induction in both J-Lat and Jurkat datasets, suggesting relatively low overlap between responses to TNF-alpha induction in Case and Control groups. To further determine the extent of TNF-alpha response overlap between case and control groups, DGE data was used to compute functional enrichment analysis with $\operatorname{StringDB}(39)$ version 11.0 with Gene Ontology (GO) framework at the Cellular Component and Biological Process levels. Highly significant (FDR $<0.01$ ) GO Cellular components enriched in J-Lat case group include the 'NF-kappaB complex', the 'spliceosomal complex', and 'secretory granule membrane', which do not overlap with the single 'cytosolic ribosome' term found in Jurkat control group (Table 1). Likewise, GO Biological Process terms do not overlap between case and control groups, except for 'NF-kappaB signaling which is present in both case and controls groups but 2-fold more enriched in the former group (Supplementary Figure 7). The activation of the NFKB complex observed in functional enrichment analysis is consistent with the highly significant $(p-a d j<0.05)$ genes found to be differentially regulated in J-Lat cells treated with TNF-alpha including: TNFAIP3, NFKBIA, BIRC2, and NFKB2 (Table 2). However, a significant fraction of the DGE hits in the J-Lat case group (including those related to NFKB complex) were also found to be highly significant in Jurkat group, underscoring the utility of our 'subtractive' approach to tease apart partially overlapping responses. The NFKB complex related genes that were found to be differentially expressed exclusively in J-Lat cells include NFKBIA and BIRC2, which were previously found via RNA-Seq to be upregulated upon latency reversal in SIV-infected ART-suppressed nonhuman primates (40). BIRC2 was also found to be a negative regulator of HIV-transcription that could be antagonized with Smac mimetics for reversal of latency (41). The robust upregulation of BIRC2 we observed in our data set despite active HIV-transcription, can be reconciled with the paradoxical role of this gene as both a positive modulator of the canonical NFKB (cNFKB) pathway and a negative modulator of the non-canonical NFKB (ncNFKB) pathway (42), with our use of TNF-alpha engaging the cNFKB pathway.

To gain further insights into the specific transcript variants or isoforms eliciting gene expression changes, we plotted the TPM values of differentially expressed isoforms (DIE) with $p$-value $<0.01$ in the J-Lat case group (Figure 4A). Hierarchical clustering shows two distinct populations, that are up- or down- regulated upon TNFalpha induction. Those isoforms also found to be differentially expressed in Jurkat control group were highlighted in purple, and genes found to be highly significant (padj<0.1) are in bold and denoted with an asterisk. As consistent with the differential gene expression data, most of the highly significant DIE isoforms are upregulated upon TNF-alpha induction, with only a single isoform of PSAT1 being downregulated in this group. The DE isoform data confirms the involvement of NFKB-complex via significant 4-fold increases in relevant NFKBIA and BIRC2 isoform TPMs upon TNF-alpha treatment. Of particular note, is the highly significant $(\mathrm{p}-\mathrm{adj}<0.1)$ paradoxical downregulation of a PSAT1 isoform given that previous studies have found this gene to be enriched during Tat-elicited cell proliferation in productive HIV infection (43) and during FOXO1-inhibition elicited latency reversal in HIV-infected CD4 T-cells (44). This paradoxical result can be reconciled by close inspection of its exon connectivity (Figure 4B), which reveals that the downregulated isoform (NM_021154.4) lacks Exon 8 and results in a variant with 6- to 7- fold lower activity compared to the standard NM_058179.4 isoform that retains this exon (45). In addition, Exon 8 contains a serine 331 residue which was shown to be phosphorylated by IKBKE, a known activator of NFKB pathway, and this modification results in a downstream activation of the serine biosynthetic pathway (SBP) to support cell proliferation (46). Besides providing a putative link between NFKB-complex and the SBP in a latency reversal context, the coupling of exon connectivity along with differential isoform expression shows the utility of full-length approaches to clarify seemingly paradoxical mechanisms of transcriptional regulation.

To further investigate changes in splicing as a response to TNF-alpha induced viral reactivation in host cells, we used the FLAIR DiffSplice module to call alternative splicing events from collapsed isoform clusters. An intron retention event between exon 3 and exon 4 in the PSD4 gene locus was found to be significantly (padj<0.05) modulated upon TNF-alpha induction in J-Lat 10.6 cells (Figure 4C). This intron retention event, which is novel and not found in UCSC or SIB databases, was uniquely found in J-Lat 10.6 Case group and results 
in a premature termination codon (PTC) which renders this transcript variant unproductive. DRIMSeq2 data was used to calculate the percent spliced in (PSI) of this intron retention event, and showed the non-productive PTC isoform was predominant in uninduced J-Lat (60\% PSI value), but upon TNF-induction, the intron retention was downregulated resulting in 65\% PSI of productive (PRO) isoform. This dynamic is concomitant with the robust induction $(\mathrm{p}$-adj $<0.1)$ of the productive XM006712392.2 PSD4 isoform upon TNF-alpha treatment as evidenced by its 2-fold increase in normalized isoform expression (Figure 4A). PSD4 belongs to a family of Pleckstrin and Sec7 domain containing proteins (PSD or EFA6), which are associated with the plasma membrane (PM) and interact with ARF6 proteins via their Sec7 guanine exchange factor (GEF) domain to regulate PM and endosomal traffic (47). ARF6 has been previously found to be a molecular determinant of HIV-1 Gag association with the PM (48) via its activation of PIP5K lipid modifying enzyme(49) which enhances PIP2 production, an acidic phospholipid which is specifically recognized by the highly basic region of HIV Matrix for anchoring into PM (50). Despite the wealth of evidence of an ARF6 interaction with Sec 7 domain containing proteins, PSD4 has not been directly associated with productive HIV infection or evaluated for its regulation via an intron retention mechanism.

In addition to host cell transcriptional correlates, our approach also captures the HIV transcriptional signature that is concomitant to TNF-alpha induced viral reactivation in J-Lat 10.6 cells. Our isoform clustering and collapse analysis across four replicates shows the capture of all canonical HIV splice sites and all multiexonic transcripts (Figure 5A). These transcripts are divided into "Completely Spliced" (i.e. 2kb), and "Incompletely Spliced" (i.e. 4kb) classes based on the presence or lack of a D4-A7 splice event. However, unlike previous approaches $(18,21)$, direct comparison of enrichment between any transcript is possible in our approach irrespective of transcript class (Figure 5B). In addition to canonical HIV isoforms, our approach showed presence of a Nef isoform lacking canonical A5-D4 exon which despite retaining complete ORF, has not been previously observed. Additionally, a completely spliced variant of Vif was observed, which despite lacking the canonical intron retention between D4-A7, still contains a complete and undisrupted ORF upstream to this site. With regards to non-coding exons 2 and 3, these are present at much lower enrichment levels compared to previous studies(21), with non-coding exon 3 being more prevalent and associated with Rev/Nef/Tat/Env transcripts, and non-coding exon 2 being less prevalent and only associated with Tat and Nef transcripts. Gene assignment was based on a two-variables, with ORF proximity to the 5' end of isoform being initial variable, followed by the presence of an undisrupted ORF. Using this system allows isoforms to be assigned to a gene unambiguously, particularly in cases of incompletely spliced transcripts containing A4 acceptors, where ORF proximity alone would impute an unproductive Rev isoform, instead of the likely productive Env/Vpu transcript. By classifying isoforms into likely expressed genes (Figure 5C) relative gene expression can be determined, with highest enriched genes being Nef, Rev and Nef accounting for $45 \%, 27 \%$ and $20 \%$ of transcripts respectively. The high abundance of Nef and Rev, compared to the relatively low level of Tat is consistent with previous studies $(21,51)$ and concordant with splice acceptor usage in our data (Figure 5D). Moreover, the relatively high abundance of Rev is consistent with the requirement of this viral protein to oligomerize on RRE substrates to ensure the export of unspliced and partially unspliced transcripts out of the nucleus (52). As expected, the D1 splice donor shows highest usage followed closely by D4, the latter of which is consistent with the highest enrichment observed in transcripts containing the D4-A7 splice junction (i.e. fully spliced) (Figure 5E). HIV splicing dynamics can be further explored with a splice junction matrix (Figure 5F), showing all observed combinations of splice donor/acceptor junctions along with their enrichment, with D1-A5 and D4-A7 junctions being the most highly expressed junctions and correlating to Env to Rev/Nef transcripts respectively. The HIV transcriptional signature revealed in our approach can be used to interrogate transcriptional changes as a response to a variety of HIV reactivation methodologies, host cell gene manipulations (i.e. knockdown and knockouts), and viral sequence manipulations, allowing greater granularity in the study of the interdependence of host and viral transcriptional regulation during viral infection. 


\section{$\underline{\text { Discussion }}$}

In this study, we introduce and validate a full-length direct cDNA sequencing pipeline for the simultaneous profiling of poly-adenylated viral and host cell transcripts from unamplified cDNA. This approach is supported by the use of two high performing reverse transcriptases and Oligo-d(T) priming, coupled to a novel one-step chemical ablation of 3' RNA ends, CASPR, which reduces rRNA reads and enriches poly-adenylated transcripts. We use this approach to simultaneously interrogate host and viral transcriptional dynamics within a full-length sequencing context in a relevant cell line model of HIV reactivation. This has allowed us to identify putative host factors of HIV transcriptional activation that contain exon skipping events (PSAT1) or novel intron retentions (PSD4). In addition, our full-length RNA-Seq pipeline is agnostic to sequencing methodology or library preparation approaches, and widely applicable for the study of viral transcription dynamics in host cells.

CASPR treatment in combination with MarathonRT were critical components in maximizing the quantitative capture of full-length host cell transcripts. The exact mechanism of CASPR-mediated improvements in obtaining full-length cDNA are beyond the scope of this manuscript; however, our data suggests that these improvements in priming specificity (via reduction of primer-independent products) are modulated by the 3'-OH ends of RNA inputs. The presence of non-specific cDNAs generated in a primer independent manner has been a largely overlooked artefact of reverse transcription. This has been cemented by the notion that exogenous DNA primers are an absolute requirement for reverse transcription, despite growing evidence of primer-independent cDNA generation in a variety of reverse transcriptases, which has been variously reported in the field as "falsepriming", "self-priming", and "background priming" (53-56). Moreover, the fact that the CASPR reagent resulted in improvements in the performance of both MRT and SSIV despite their different origins, and in a variety of RNA inputs and priming modalities, points to RT initiation in absence of exogenous primer being a prevalent phenomenon. Primer-independent cDNA products are also a barrier in the study of replication dynamics of other RNA viruses where expression of negative strand intermediate transcripts is a hallmark of active viral replication, as is the case in Dengue Virus, West Nile Virus, Hepatitis C Virus, SARS-CoV2 and others (55,57-60). This suggests wide applicability of the CASPR reagent which, coupled with a suitable priming modality and a processive RT, could increase the breadth and sensitivity in the capture of full-length transcripts of interest in other relevant systems.

Given the polycistronic nature of HIV RNA, the full exon connectivity provided by this pipeline is a critical component in the unambiguous assignment of detected isoforms to a likely expressed gene or in the identification of novel splice junctions. This is not a minor a problem for HIV, where a single intron retention event between two isoforms with seemingly identical splice junctions could result in expression of another viral gene. Full-length reads obtained in our pipeline allow straightforward isoform assignment and productivity analysis for the majority of HIV genes. However, the case of partially unspliced transcripts containing A4 or A5 splice sites constitutes an illustrative case where gene assignment can remain ambiguous even with full-length isoform information. Based on the premise that the closest ORF to the 5' end of transcript constitutes the determinant factor in gene is expressed, partially unspliced isoforms containing A4 sites would translate to a unproductive Rev (since the CDS is disrupted by the D4/A7 intron retention), whereas those containing A5 would be translated as productive Env/Vpu. This ambiguity, however, is consistent with previous studies showing HIV co-opts the host cell translation machinery in non-canonical ways to further regulate its gene expression via leaky ribosomal scanning or ribosome shunting (61). Thus, ORF proximity to 5' end is a necessary but not sufficient factor in determining which gene is eventually expressed from a particular splice variant. In these cases, we used the presence of a complete and non-disrupted CDS as a second prioritization scheme for gene assignment, whereby a partially spliced variant containing an A4 junction is likely to code for productive Env/Vpu and not an unproductive Rev (i.e., prioritization of longest ORF). Given the dynamic nature of HIV RNA secondary structure proximal to splice junctions(62) and its inhibitory role in ribosome scanning, future studies coupling splice variant detection with DMS-MaP secondary structure probing(23) might provide additional clarity on Rev and Env/Vpu translational regulation, while allowing additional variables for consideration of gene assignment and productivity analyses.

Despite the moderate sequencing depth used in our studies, the yield and coverage increases elicited by CASPR allowed sufficient capture of host cell transcript variants for biologically meaningful DGE/DIE analyses while also detecting all canonical splice junctions in HIV isoforms. Sequencing throughput in our studies was a 
function of the MinION sequencer used, which allowed for rapid method development and validation studies at the expense of number of reads (compared to some large scale transcriptomic studies of rare AS transcripts) (31). Any throughput constraints, can be easily addressed in future studies by adopting higher throughput platforms available from ONT, including the GridION and PromethION each with five- and 250-fold higher throughput. An additional consideration in our platform hinges on the number of cells required for dispensing with PCR amplification, currently 50,000 cells are required to obtain sufficient total RNA. The required number of cells might not be unreasonable when using cultured cell lines, but when using primary cells or clinical samples, the requirement might be a limitation without further PCR amplification. For these types of samples, a cDNA amplification library preparation kit which attaches 5' and 3' adapters during RT can be used with CASPR-treated RNA inputs, followed by emulsion PCR with a single primer set and with a modest number of cycles to minimize PCR sampling bias (63), and allow for enrichment comparison between transcripts.

An interesting finding revealed by our study is the predominant intron retention event observed in the PSD4 locus of uninduced J-Lat cells, which results in expression of a truncated and inactive isoform due to a premature termination codon. The biological relevance of this AS event is not yet established; however, the role of other Sec7 domain containing proteins in targeting of viral components to the plasma membrane via its GEF activity and interaction with ARF6 has been thoroughly documented (49). The reduction in expression of productive PSD4 could reduce the amount of active ARF6 and thus affect the balance of phosphatidylinositol that allows permissive assembly or entry of viral components proximal to the plasma membrane. However, intron retention events are widespread in cancer transcriptomes (64), and given the origin of J-Lat 10.6 cells from immortalized T-cell leukemia PBMCs, the causal relationship between the modulation of PSD4 (and other AS isoforms) and HIV replicative capacity has to be thoroughly validated in primary cells.

In summary, we developed and systematically validated a full-length RNA-seq pipeline for assessing viral RNA transcript dynamics within a host cell transcriptome. This approach is supported by use of highly processive RTs, coupled with CASPR, a novel one-step CDS enrichment strategy that outperforms prevailing PolyA+ selection strategies in the breadth and sensitivity of capture of host cell and HIV transcripts. An initial assessment using our developed technology has allowed identification of putative host factors that affect HIV transcriptional activation, which provides a framework for further studies of differential regulation of host cell transcripts and their associated HIV transcriptional signature. We expect this pipeline will provide greater insights into the dynamics that affect viral activation within host cells and its associated HIV transcriptional state, while also being suitable for the study of transcriptional regulation in infections with other RNA viruses.

\section{FUNDING}

National Human Genome Research Institute [R01HG009622 to B.E.T.]; National Institute of Allergy and Infectious Diseases [U54AI150472 to B.E.T]

\section{DATA AVAILABILITY}

Sequencing data has been submitted to the NCBI Sequence Read Archive (SRA) under BioProject ID PRJNA801353

\section{CONFLICT OF INTEREST STATEMENT}

CMG and BET are listed as inventors on a provisional patent filed by Seattle Children's Research Institute related to the CASPR methodology. 


\section{$\underline{\text { References }}$}

1. Pan, Q., Shai, O., Lee, L.J., Frey, B.J. and Blencowe, B.J. (2008) Deep surveying of alternative splicing complexity in the human transcriptome by high-throughput sequencing. Nat Genet, 40, 1413-1415.

2. Fu, X.D. and Ares, M., Jr. (2014) Context-dependent control of alternative splicing by RNA-binding proteins. Nat Rev Genet, 15, 689-701.

3. Nilsen, T.W. and Graveley, B.R. (2010) Expansion of the eukaryotic proteome by alternative splicing. Nature, 463, 457-463.

4. Liu, Y., Gonzàlez-Porta, M., Santos, S., Brazma, A., Marioni, J.C., Aebersold, R., Venkitaraman, A.R. and Wickramasinghe, V.O. (2017) Impact of Alternative Splicing on the Human Proteome. Cell Rep, 20, 12291241.

5. Wang, E.T., Sandberg, R., Luo, S., Khrebtukova, I., Zhang, L., Mayr, C., Kingsmore, S.F., Schroth, G.P. and Burge, C.B. (2008) Alternative isoform regulation in human tissue transcriptomes. Nature, 456, 470-476.

6. Park, E., Pan, Z., Zhang, Z., Lin, L. and Xing, Y. (2018) The Expanding Landscape of Alternative Splicing Variation in Human Populations. Am J Hum Genet, 102, 11-26.

7. Ashraf, U., Benoit-Pilven, C., Lacroix, V., Navratil, V. and Naffakh, N. (2019) Advances in Analyzing VirusInduced Alterations of Host Cell Splicing. Trends Microbiol, 27, 268-281.

8. Imbeault, M., Giguère, K., Ouellet, M. and Tremblay, M.J. (2012) Exon level transcriptomic profiling of HIV-1-infected CD4(+) T cells reveals virus-induced genes and host environment favorable for viral replication. PLoS Pathog, 8, e1002861.

9. Byun, S., Han, S., Zheng, Y., Planelles, V. and Lee, Y. (2020) The landscape of alternative splicing in HIV-1 infected CD4 T-cells. BMC Med Genomics, 13, 38.

10. Wojcechowskyj, J.A., Didigu, C.A., Lee, J.Y., Parrish, N.F., Sinha, R., Hahn, B.H., Bushman, F.D., Jensen, S.T., Seeholzer, S.H. and Doms, R.W. (2013) Quantitative phosphoproteomics reveals extensive cellular reprogramming during HIV-1 entry. Cell Host Microbe, 13, 613-623.

11. Pabis, M., Corsini, L., Vincendeau, M., Tripsianes, K., Gibson, T.J., Brack-Werner, R. and Sattler, M. (2019) Modulation of HIV-1 gene expression by binding of a ULM motif in the Rev protein to UHM-containing splicing factors. Nucleic Acids Res, 47, 4859-4871.

12. Lapek, J.D., Jr., Lewinski, M.K., Wozniak, J.M., Guatelli, J. and Gonzalez, D.J. (2017) Quantitative Temporal Viromics of an Inducible HIV-1 Model Yields Insight to Global Host Targets and Phospho-Dynamics Associated with Protein Vpr. Mol Cell Proteomics, 16, 1447-1461.

13. Mueller, N., Pasternak, A.O., Klaver, B., Cornelissen, M., Berkhout, B. and Das, A.T. (2018) The HIV-1 Tat Protein Enhances Splicing at the Major Splice Donor Site. J Virol, 92.

14. Zhou, C., Liu, S., Song, W., Luo, S., Meng, G., Yang, C., Yang, H., Ma, J., Wang, L., Gao, S. et al. (2018) Characterization of viral RNA splicing using whole-transcriptome datasets from host species. Scientific Reports, 8, 3273.

15. Karn, J. and Stoltzfus, C.M. (2012) Transcriptional and posttranscriptional regulation of HIV-1 gene expression. Cold Spring Harb Perspect Med, 2, a006916.

16. Kutluay, S.B., Emery, A., Penumutchu, S.R., Townsend, D., Tenneti, K., Madison, M.K., Stukenbroeker, A.M., Powell, C., Jannain, D., Tolbert, B.S. et al. (2019) Genome-Wide Analysis of Heterogeneous Nuclear Ribonucleoprotein (hnRNP) Binding to HIV-1 RNA Reveals a Key Role for hnRNP H1 in Alternative Viral mRNA Splicing. J Virol, 93.

17. Esquiaqui, J.M., Kharytonchyk, S., Drucker, D. and Telesnitsky, A. (2020) HIV-1 spliced RNAs display transcription start site bias. Rna, 26, 708-714.

18. Emery, A., Zhou, S., Pollom, E. and Swanstrom, R. (2017) Characterizing HIV-1 Splicing by Using NextGeneration Sequencing. J Virol, 91.

19. Shi, H., Zhou, Y., Jia, E., Pan, M., Bai, Y. and Ge, Q. (2021) Bias in RNA-seq Library Preparation: Current Challenges and Solutions. BioMed Research International, 2021, 6647597. 
20. Byrne, A., Beaudin, A.E., Olsen, H.E., Jain, M., Cole, C., Palmer, T., DuBois, R.M., Forsberg, E.C., Akeson, M. and Vollmers, C. (2017) Nanopore long-read RNAseq reveals widespread transcriptional variation among the surface receptors of individual B cells. Nature communications, 8, 16027-16027.

21. Ocwieja, K.E., Sherrill-Mix, S., Mukherjee, R., Custers-Allen, R., David, P., Brown, M., Wang, S., Link, D.R., Olson, J., Travers, K. et al. (2012) Dynamic regulation of HIV-1 mRNA populations analyzed by singlemolecule enrichment and long-read sequencing. Nucleic Acids Res, 40, 10345-10355.

22. Nguyen Quang, N., Goudey, S., Ségéral, E., Mohammad, A., Lemoine, S., Blugeon, C., Versapuech, M., Paillart, J.-C., Berlioz-Torrent, C., Emiliani, S. et al. (2020) Dynamic nanopore long-read sequencing analysis of HIV-1 splicing events during the early steps of infection. Retrovirology, 17, 25.

23. Guo, L.T., Adams, R.L., Wan, H., Huston, N.C., Potapova, O., Olson, S., Gallardo, C.M., Graveley, B.R., Torbett, B.E. and Pyle, A.M. (2020) Sequencing and Structure Probing of Long RNAs Using MarathonRT: A Next-Generation Reverse Transcriptase. J Mol Biol, 432, 3338-3352.

24. Zhao, C., Liu, F. and Pyle, A.M. (2018) An ultraprocessive, accurate reverse transcriptase encoded by a metazoan group II intron. Rna, 24, 183-195.

25. Jordan, A., Bisgrove, D. and Verdin, E. (2003) HIV reproducibly establishes a latent infection after acute infection of T cells in vitro. The EMBO journal, 22, 1868-1877.

26. Spina, C.A., Anderson, J., Archin, N.M., Bosque, A., Chan, J., Famiglietti, M., Greene, W.C., Kashuba, A., Lewin, S.R., Margolis, D.M. et al. (2013) An in-depth comparison of latent HIV-1 reactivation in multiple cell model systems and resting CD4+ T cells from aviremic patients. PLoS Pathog, 9, e1003834.

27. Weiss, A., Wiskocil, R. and Stobo, J. (1984) The role of T3 surface molecules in the activation of human T cells: a two-stimulus requirement for IL 2 production reflects events occurring at a pre-translational level. The Journal of Immunology, 133, 123-128.

28. Gubler, U. and Hoffman, B.J. (1983) A simple and very efficient method for generating cDNA libraries. Gene, 25, 263-269.

29. Li, H., Handsaker, B., Wysoker, A., Fennell, T., Ruan, J., Homer, N., Marth, G., Abecasis, G., Durbin, R. and Subgroup, G.P.D.P. (2009) The Sequence Alignment/Map format and SAMtools. Bioinformatics, 25, 2078-2079.

30. Wang, L., Nie, J., Sicotte, H., Li, Y., Eckel-Passow, J.E., Dasari, S., Vedell, P.T., Barman, P., Wang, L., Weinshiboum, R. et al. (2016) Measure transcript integrity using RNA-seq data. BMC Bioinformatics, 17, 58.

31. Tang, A.D., Soulette, C.M., van Baren, M.J., Hart, K., Hrabeta-Robinson, E., Wu, C.J. and Brooks, A.N. (2020) Full-length transcript characterization of SF3B1 mutation in chronic lymphocytic leukemia reveals downregulation of retained introns. Nat Commun, 11, 1438.

32. Pertea, G. and Pertea, M. (2020) GFF Utilities: GffRead and GffCompare [version 1; peer review: 3 approved]. F1000Research, 9.

33. Ståhlberg, A., Kubista, M. and Pfaffl, M. (2004) Comparison of Reverse Transcriptases in Gene Expression Analysis. Clinical Chemistry, 50, 1678-1680.

34. Zucha, D., Androvic, P., Kubista, M. and Valihrach, L. (2019) Performance Comparison of Reverse Transcriptases for Single-Cell Studies. Clinical Chemistry, 66, 217-228.

35. O'Neil, D., Glowatz, H. and Schlumpberger, M. (2013) Ribosomal RNA depletion for efficient use of RNAseq capacity. Curr Protoc Mol Biol, Chapter 4, Unit 419.

36. Zhao, S., Zhang, Y., Gamini, R., Zhang, B. and von Schack, D. (2018) Evaluation of two main RNA-seq approaches for gene quantification in clinical RNA sequencing: polyA+ selection versus rRNA depletion. Sci Rep, 8, 4781.

37. Hardwick, S.A., Deveson, I.W. and Mercer, T.R. (2017) Reference standards for next-generation sequencing. Nature Reviews Genetics, 18, 473-484.

38. Watts, J.M., Dang, K.K., Gorelick, R.J., Leonard, C.W., Bess, J.W., Jr., Swanstrom, R., Burch, C.L. and Weeks, K.M. (2009) Architecture and secondary structure of an entire HIV-1 RNA genome. Nature, 460, 711-716. 
39. Szklarczyk, D., Gable, A.L., Lyon, D., Junge, A., Wyder, S., Huerta-Cepas, J., Simonovic, M., Doncheva, N.T., Morris, J.H. and Bork, P. (2019) STRING v11: protein-protein association networks with increased coverage, supporting functional discovery in genome-wide experimental datasets. Nucleic acids research, 47, D607-D613.

40. Nixon, C.C., Mavigner, M., Sampey, G.C., Brooks, A.D., Spagnuolo, R.A., Irlbeck, D.M., Mattingly, C., Ho, P.T., Schoof, N., Cammon, C.G. et al. (2020) Systemic HIV and SIV latency reversal via non-canonical NFKB signalling in vivo. Nature, 578, 160-165.

41. Pache, L., Dutra, M.S., Spivak, A.M., Marlett, J.M., Murry, J.P., Hwang, Y., Maestre, A.M., Manganaro, L., Vamos, M., Teriete, P. et al. (2015) BIRC2/cIAP1 Is a Negative Regulator of HIV-1 Transcription and Can Be Targeted by Smac Mimetics to Promote Reversal of Viral Latency. Cell Host Microbe, 18, 345-353.

42. Hrdinka, M. and Yabal, M. (2019) Inhibitor of apoptosis proteins in human health and disease. Genes Immun, 20, 641-650.

43. Jarboui, M.A., Bidoia, C., Woods, E., Roe, B., Wynne, K., Elia, G., Hall, W.W. and Gautier, V.W. (2012) Nucleolar protein trafficking in response to HIV-1 Tat: rewiring the nucleolus. PLoS One, 7, e48702.

44. Vallejo-Gracia, A., Chen, I.P., Perrone, R., Besnard, E., Boehm, D., Battivelli, E., Tezil, T., Krey, K., Raymond, K.A., Hull, P.A. et al. (2020) FOXO1 promotes HIV latency by suppressing ER stress in T cells. Nat Microbiol.

45. Baek, J.Y., Jun, D.Y., Taub, D. and Kim, Y.H. (2003) Characterization of human phosphoserine aminotransferase involved in the phosphorylated pathway of L-serine biosynthesis. Biochem J, 373, 191200.

46. Xu, R., Jones, W., Wilcz-Villega, E., Costa, A.S., Rajeeve, V., Bentham, R.B., Bryson, K., Nagano, A., Yaman, B., Olendo Barasa, S. et al. (2020) The breast cancer oncogene IKKE coordinates mitochondrial function and serine metabolism. EMBO reports, 21, e48260.

47. Sztul, E., Chen, P.W., Casanova, J.E., Cherfils, J., Dacks, J.B., Lambright, D.G., Lee, F.S., Randazzo, P.A., Santy, L.C., Schürmann, A. et al. (2019) ARF GTPases and their GEFs and GAPs: concepts and challenges. Mol Biol Cell, 30, 1249-1271.

48. Chukkapalli, V. and Ono, A. (2011) Molecular determinants that regulate plasma membrane association of HIV-1 Gag. J Mol Biol, 410, 512-524.

49. Van Acker, T., Tavernier, J. and Peelman, F. (2019) The Small GTPase Arf6: An Overview of Its Mechanisms of Action and of Its Role in Host ${ }^{-}$Pathogen Interactions and Innate Immunity. Int J Mol Sci, 20.

50. Freed, E.O. (2006) HIV-1 Gag: flipped out for PI(4,5)P(2). Proc Natl Acad Sci U S A, 103, 11101-11102.

51. Erkelenz, S., Hillebrand, F., Widera, M., Theiss, S., Fayyaz, A., Degrandi, D., Pfeffer, K. and Schaal, H. (2015) Balanced splicing at the Tat-specific HIV-1 3'ss A3 is critical for HIV-1 replication. Retrovirology, 12, 29.

52. Fernandes, J., Jayaraman, B. and Frankel, A. (2012) The HIV-1 Rev response element. RNA Biology, 9, 611.

53. Lanford, R.E., Chavez, D., Chisari, F.V. and Sureau, C. (1995) Lack of detection of negative-strand hepatitis $C$ virus RNA in peripheral blood mononuclear cells and other extrahepatic tissues by the highly strandspecific $r$ Tth reverse transcriptase PCR. J Virol, 69, 8079-8083.

54. Haddad, F., Qin, A.X., Giger, J.M., Guo, H. and Baldwin, K.M. (2007) Potential pitfalls in the accuracy of analysis of natural sense-antisense RNA pairs by reverse transcription-PCR. BMC Biotechnol, 7, 21.

55. Tuiskunen, A., Leparc-Goffart, I., Boubis, L., Monteil, V., Klingström, J., Tolou, H.J., Lundkvist, A. and Plumet, S. (2010) Self-priming of reverse transcriptase impairs strand-specific detection of dengue virus RNA. J Gen Virol, 91, 1019-1027.

56. Frech, B. and Peterhans, E. (1994) RT-PCR: 'background priming' during reverse transcription. Nucleic Acids Res, 22, 4342-4343.

57. Lim, S.M., Koraka, P., Osterhaus, A.D. and Martina, B.E. (2013) Development of a strand-specific realtime qRT-PCR for the accurate detection and quantitation of West Nile virus RNA. J Virol Methods, 194, 146-153.

58. Lerat, H., Berby, F., Trabaud, M.A., Vidalin, O., Major, M., Trépo, C. and Inchauspé, G. (1996) Specific detection of hepatitis C virus minus strand RNA in hematopoietic cells. J Clin Invest, 97, 845-851. 
59. Fehr, A.R. and Perlman, S. (2015) Coronaviruses: an overview of their replication and pathogenesis. Methods Mol Biol, 1282, 1-23.

60. Sawicki, S.G. (2008) Coronavirus Genome Replication. Viral Genome Replication, 25-39.

61. Guerrero, S., Batisse, J., Libre, C., Bernacchi, S., Marquet, R. and Paillart, J.C. (2015) HIV-1 replication and the cellular eukaryotic translation apparatus. Viruses, 7, 199-218.

62. Tomezsko, P.J., Corbin, V.D.A., Gupta, P., Swaminathan, H., Glasgow, M., Persad, S., Edwards, M.D., McIntosh, L., Papenfuss, A.T., Emery, A. et al. (2020) Determination of RNA structural diversity and its role in HIV-1 RNA splicing. Nature, 582, 438-442.

63. Gallardo, C.M., Wang, S., Montiel-Garcia, D.J., Little, S.J., Smith, D.M., Routh, A.L. and Torbett, B.E. (2021) MrHAMER yields highly accurate single molecule viral sequences enabling analysis of intra-host evolution. Nucleic Acids Res, 49, e70.

64. Dvinge, H. and Bradley, R.K. (2015) Widespread intron retention diversifies most cancer transcriptomes. Genome Med, 7, 45. 


\section{Figures and Tables}

A

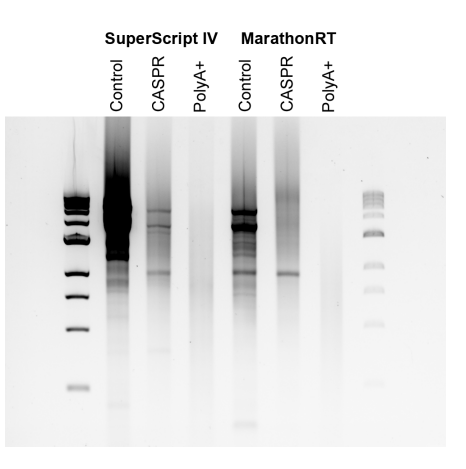

B

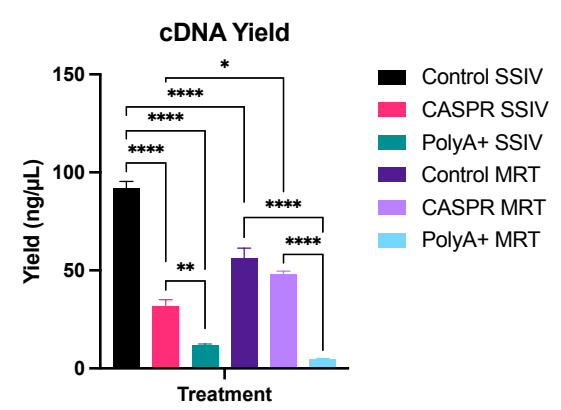

C

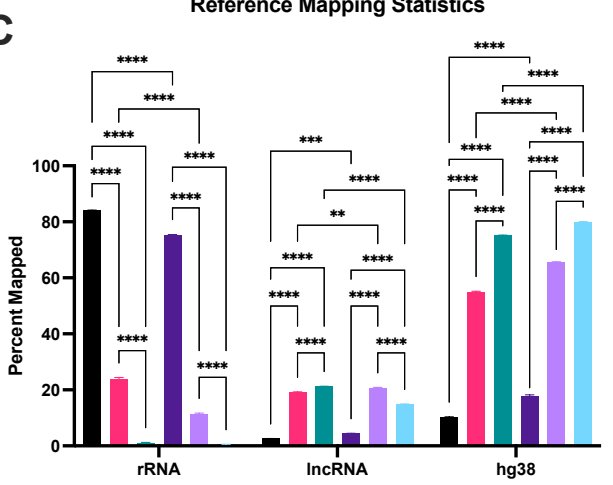

D

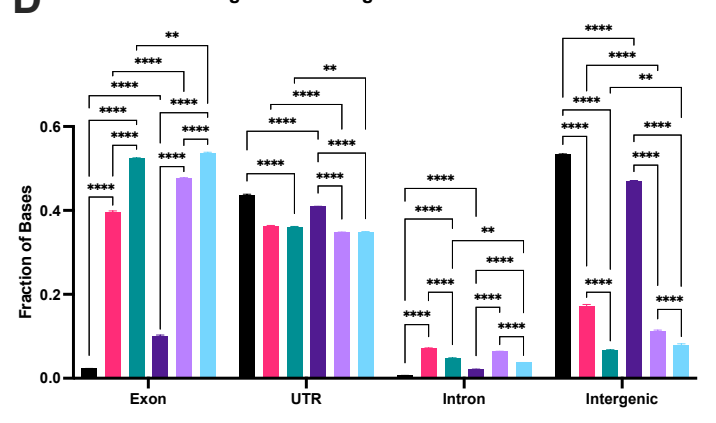

E

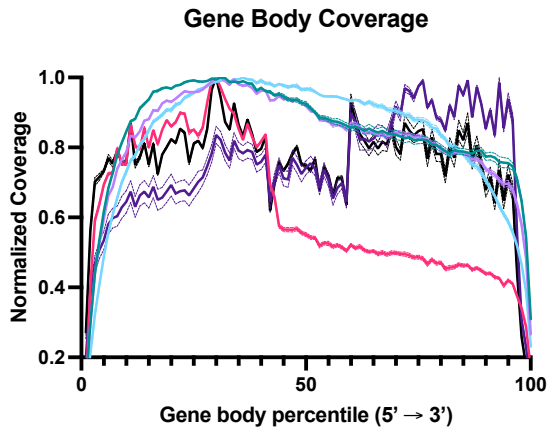

$\mathbf{F}$

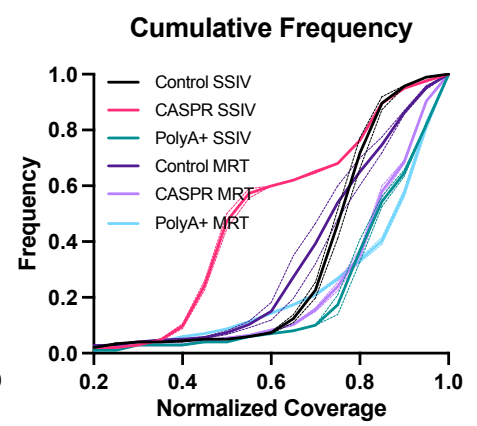

Figure 1 - CASPR improves the specificity of Oligo-d(T) primed RT when using total RNA inputs by reducing rRNA and increasing coverage evenness of protein-coding transcripts.

(A) Agarose gel electrophoresis of double stranded cDNA products that were Reverse Transcribed with Oligo$\mathrm{d}(\mathrm{T})$ priming with SSIV or MRT with no CDS enrichment (Control), CASPR or PolyA+ selection. (B) cDNA yield of different RT and CDS enrichment combinations as measured spectrophotometrically. (C) Fraction of reads uniquely mapped to the listed references using Nanopore sequencing. (D) Intragenic and Intergenic reads distributions. (E) Gene body coverage of protein coding transcripts, and (F) Cumulative Frequency distribution of Gene Body Coverage. All values are means \pm SEM. Statistical significance calculated with two-way ANOVA with Tukey multiple comparisons test, $\mathrm{p}<0.05(*), \mathrm{p}<0.01(* *), \mathrm{p}<0.001(* * *), \mathrm{p}<0.0001(* * * *)$ 

available under aCC-BY-NC-ND 4.0 International license.

A

SIRV Mapping Percentage

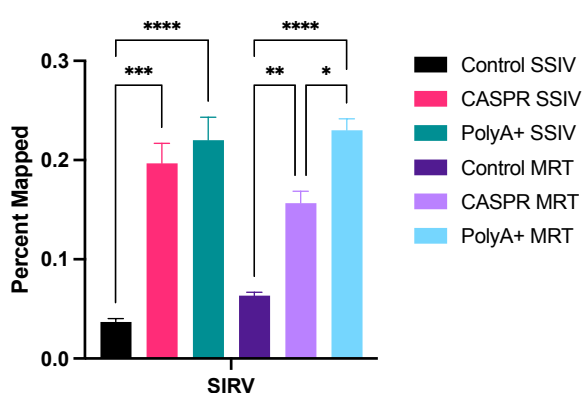

B

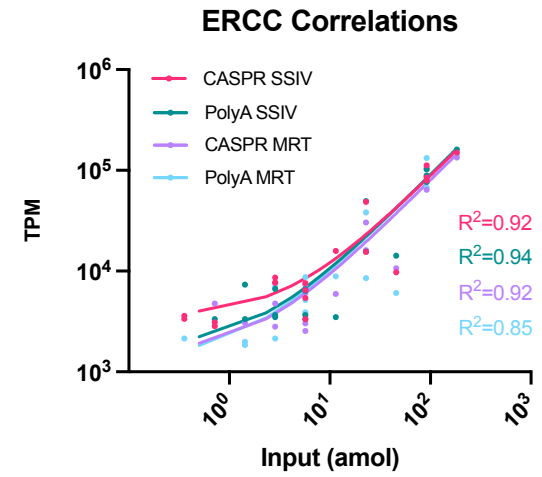

C
D

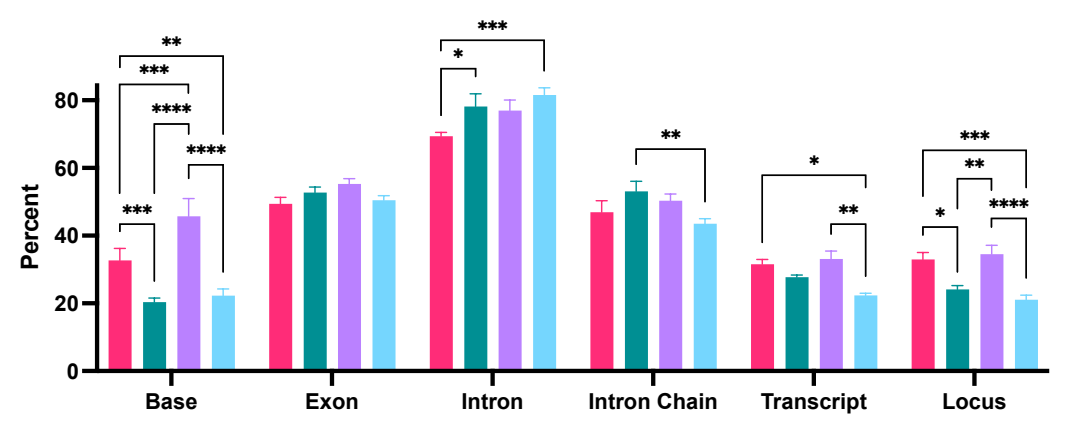

$E$
Gene expression correlations (TPM)
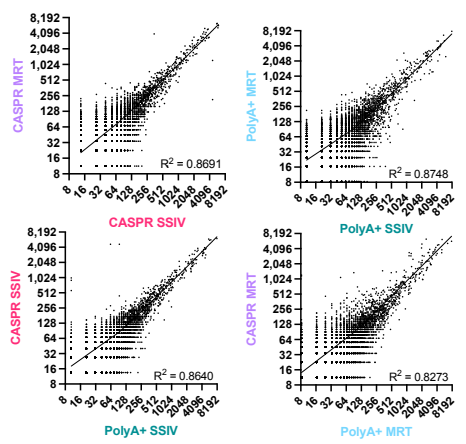

E

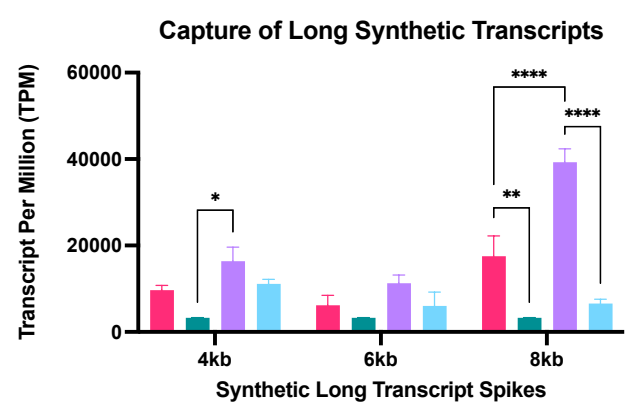

F

Transcript Model

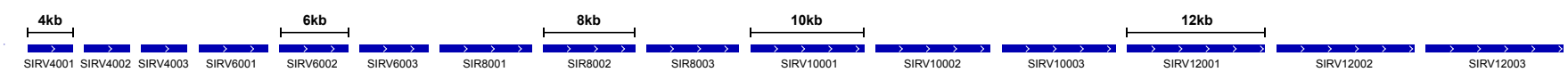

Coverage

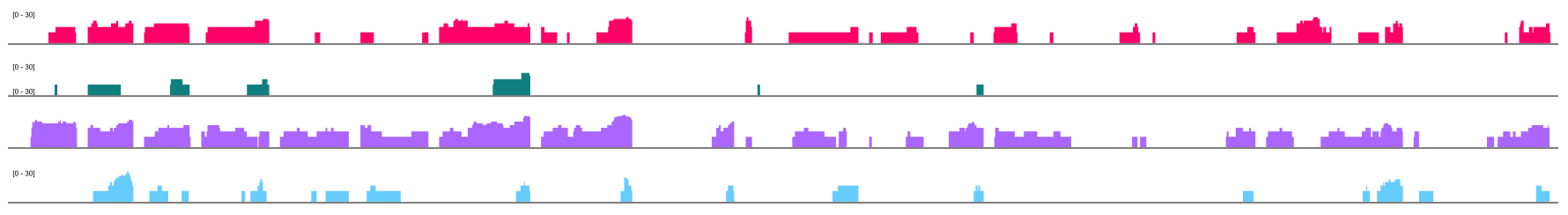

Figure 2 - Validation with synthetic reference standards show CASPR is functionally equivalent to PolyA+ selection, but results in higher cDNA yield, coverage evenness and capture of long transcripts.

(A) Percent of reads uniquely mapped to SIRV reference sequences. (B) Correlations of gene expression TPM values with absolute input amounts of each synthetic transcript (in attomoles) for ERCC subsets. (C) Hg38 gene expression correlations between different RTs and CDS enrichment strategies. (D) Transcript Discovery Sensitivity calculation using FLAIR-derived transcriptome and hg38 gtf annotation file. (E) Efficiency of capture of Long SIRVs of $4 \mathrm{~kb}, 6 \mathrm{~kb}$, and $8 \mathrm{~kb}$ size classes. (F) Raw coverage visualized via IGV of all Long SIRVs for each RT and CDS enrichment strategy combination. All samples ran in triplicate $(\mathrm{n}=3)$. All values are Means \pm SEM. Statistical significance calculated with two-way ANOVA with Tukey multiple comparisons test, $\mathrm{p}<0.05(*)$, $\mathrm{p}<0.01(* *), \mathrm{p}<0.001(* * *), \mathrm{p}<0.0001(* * * *)$ 
A

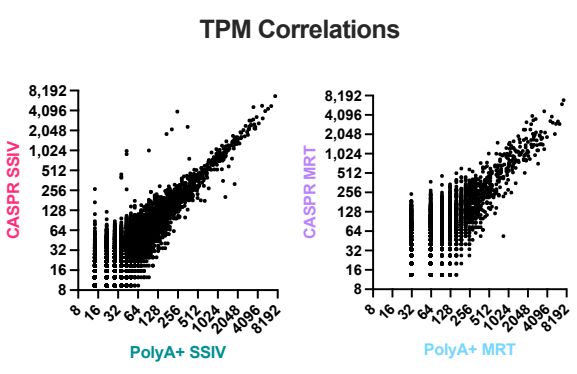

B

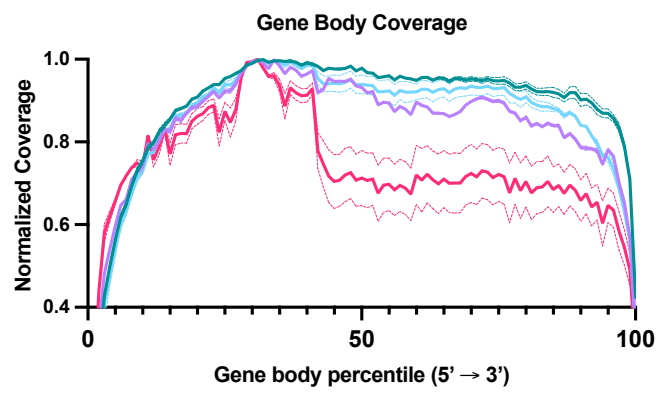

C

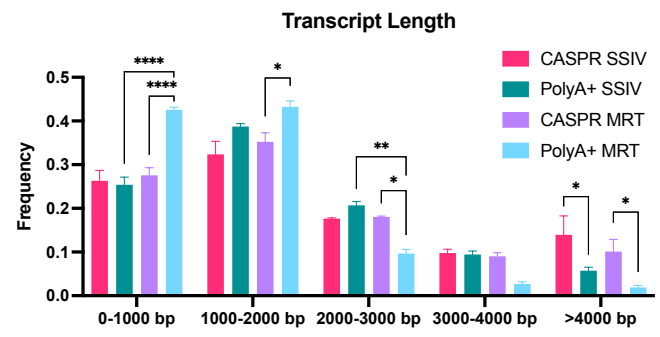

D

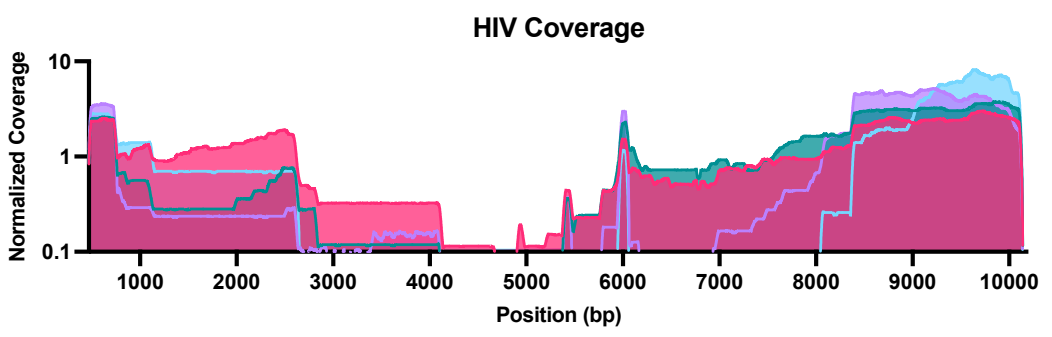

E

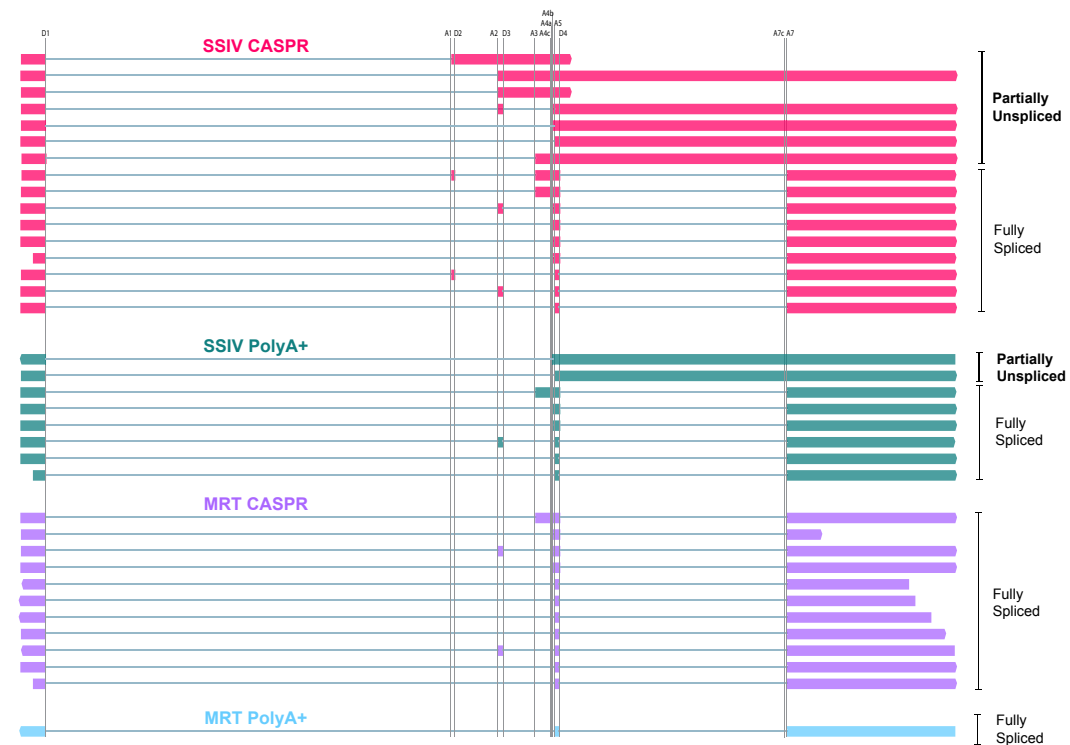

Figure 3 - Evaluation of RT conditions and CDS enrichment strategies in capture of host and viral transcripts in cell line actively expressing HIV

(A) Gene expression correlations for each CDS enrichment strategy when using SSIV and MRT. (B) Gene-Body coverage of protein coding hg38 transcripts. (C) Frequency of transcript lengths derived from FLAIR isoform analysis pipeline, binned at 100bp intervals. (D) Coverage Map of raw reads. All samples ran in duplicate $(n=2)$. (E) Visualization of isoform structure of multiexonic HIV transcripts processed with Pinfish pipeline. All values are means \pm SEM. Statistical significance calculated with two-way ANOVA with Tukey multiple comparisons test, $\mathrm{p}<0.05(*), \mathrm{p}<0.01(* *), \mathrm{p}<0.001(* * *), \mathrm{p}<0.0001(* * * *)$ 
bioRxiv preprint doi: https://doi.org/10.1101/2022.01.27.478099; this version posted February 2, 2022. The copyright holder for this preprint (which was not certified by peer review) is the author/funder, who has granted bioRxiv a license to display the preprint in perpetuity. It is made available under aCC-BY-NC-ND 4.0 International license.

Table 1 - Significant functional enrichments elicited by TNF-alpha

\begin{tabular}{|lllllll|}
\hline \#term ID & term description & $\begin{array}{l}\text { genes } \\
\text { mapped }\end{array}$ & $\begin{array}{l}\text { enrichment } \\
\text { score }\end{array}$ & FDR & method & Sample Group \\
\hline GO:0033256 & I-kappaB/NF-kappaB complex & 4 & 5.53683 & 0.00055 & afc & J-Lat (Case) \\
GO:0005681 & spliceosomal complex & 112 & 1.24205 & 0.00074 & ks & J-Lat (Case) \\
GO:0030667 & secretory granule membrane & 59 & 0.566506 & 0.0032 & ks & J-Lat (Case) \\
GO:0022626 & cytosolic ribosome & 68 & 0.22662 & 0.00054 & ks & Jurkat (Control) \\
\hline
\end{tabular}

Table 2 - Differential Gene Expression in J-Lat 10.6 case group elicited by TNF-alpha

\begin{tabular}{|c|c|c|c|c|c|c|}
\hline Gene Name & baseMean & $\log 2 F C$ & IfcSE & stat & pvalue & padj \\
\hline TNFAIP3* & 28.3868218 & 2.02400656 & 0.29780197 & 6.79648477 & $1.07 \mathrm{E}-11$ & 1.76E-08 \\
\hline LIMD2* & 23.2264768 & 1.82090609 & 0.26816954 & 6.79013023 & 1.12E-11 & 1.76E-08 \\
\hline NFKBIA* & 14.644418 & 1.98383297 & 0.33138767 & 5.98644168 & 2.14E-09 & 2.24E-06 \\
\hline BIRC2* & 15.909459 & 1.83011777 & 0.31036165 & 5.89672651 & 3.71E-09 & 2.91E-06 \\
\hline MYO7B* & 251.477162 & -0.7096294 & 0.14296654 & -4.9636048 & 6.92E-07 & 0.00043442 \\
\hline FBLN2* & 37.7849837 & 0.91500472 & 0.21341341 & 4.28747525 & 1.81E-05 & 0.00945443 \\
\hline NFKB2* & 14.0678839 & 1.456552 & 0.35152177 & 4.14356126 & 3.42E-05 & 0.01533418 \\
\hline TRAF4* & 24.2017374 & 1.01974781 & 0.24850738 & 4.10349112 & 4.0696E-05 & 0.01596817 \\
\hline RNASEK ${ }^{*}$ & 64.4095627 & 0.68541206 & 0.18244994 & 3.75671294 & 1.72E-04 & 0.06004551 \\
\hline SSBP2 & 45.5283287 & 0.67051415 & 0.18678805 & 3.58970585 & 3.31E-04 & 0.10391701 \\
\hline SNRPF & 81.0650994 & -0.4868181 & 0.1521773 & -3.1990193 & 0.00137896 & 0.39350485 \\
\hline MKRN1 & 19.8006254 & 0.80917304 & 0.25865616 & 3.12837331 & 0.00175777 & 0.45980274 \\
\hline NFKB1 & 16.1716837 & 0.9648365 & 0.31784879 & 3.03552043 & 0.00240121 & 0.57979975 \\
\hline EFEMP1 & 12.5502742 & 0.92787868 & 0.31235625 & 2.97057829 & 0.0029724 & 0.61028546 \\
\hline $\mathrm{ABCC} 1$ & 29.8451097 & 0.65128555 & 0.21967884 & 2.96471677 & 0.00302962 & 0.61028546 \\
\hline PSAT1 & 47.7234616 & -0.6819414 & 0.2320121 & -2.9392494 & $3.29 E-03$ & 0.61028546 \\
\hline LSM7 & 33.4370785 & -0.5771839 & 0.19914812 & -2.8982645 & 0.00375234 & 0.61028546 \\
\hline EIF1 & 189.997178 & -0.3583028 & 0.12546079 & -2.8558944 & 0.00429158 & 0.61028546 \\
\hline TNIP1 & 20.3255463 & 0.88255174 & 0.30903108 & 2.85586724 & 0.00429195 & 0.61028546 \\
\hline HES4 & 40.1303992 & -0.6558696 & 0.23071111 & -2.8428174 & 0.00447167 & 0.61028546 \\
\hline t contr & $x^{2}$ & 50 & -5 & 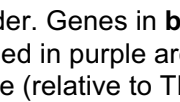 & alpha tre & $\begin{array}{l}\text { have a p- } \\
\text { pressed in }\end{array}$ \\
\hline
\end{tabular}


bioRxiv preprint doi: https://doi.org/10.1101/2022.01.27.478099; this version posted February 2, 2022. The copyright holder for this preprint (which was not certified by peer review) is the author/funder, who has granted bioRxiv a license to display the preprint in perpetuity. It is made available under aCC-BY-NC-ND 4.0 International license.

A

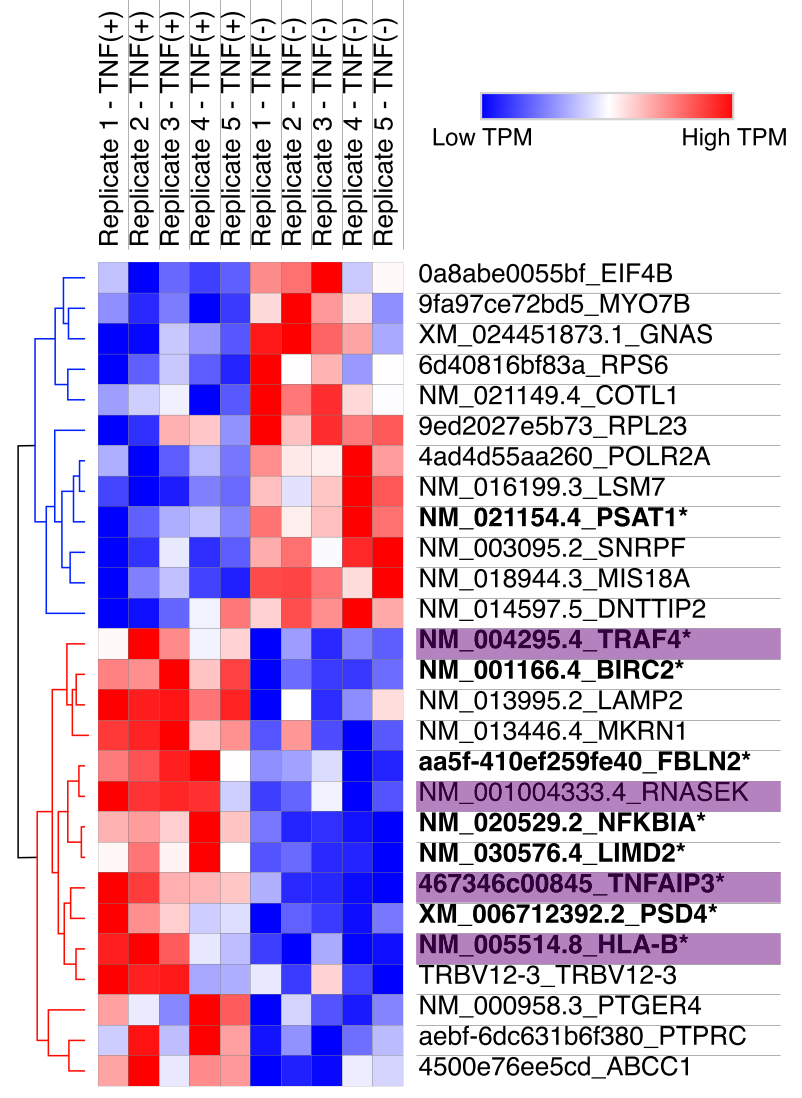

B

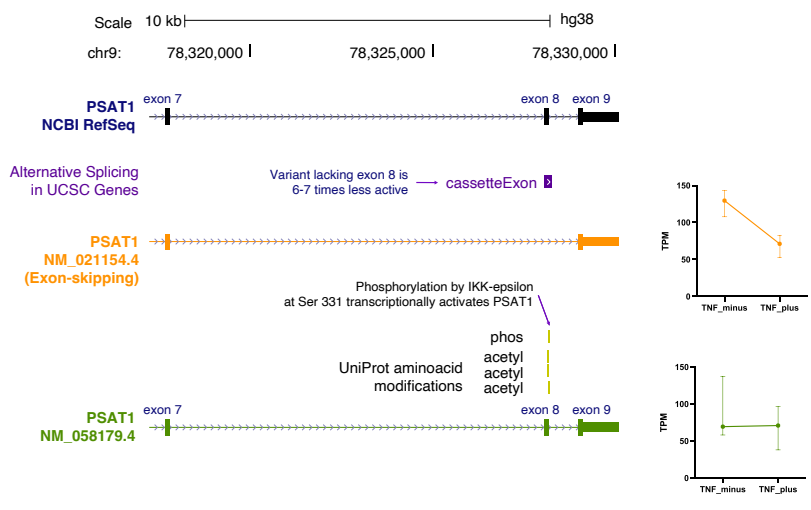

C

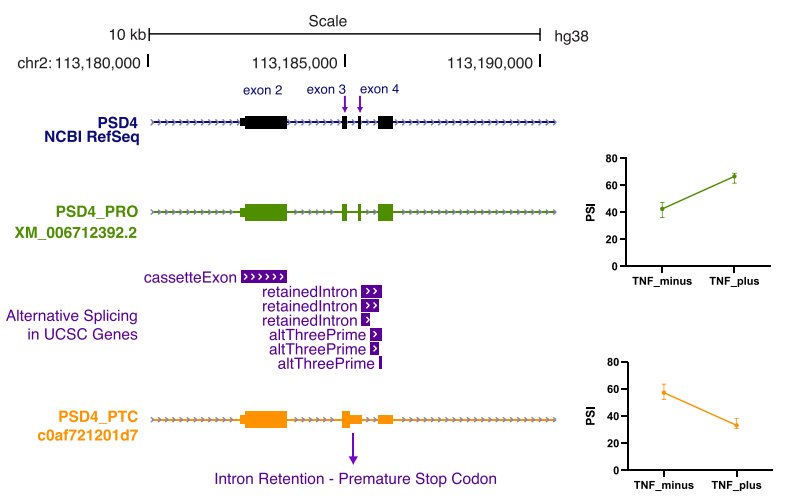

Figure 4 - Differential Isoform Expression analysis shows putative HIV host factors PSAT1 and PSD4 are alternatively spliced in host cells upon HIV reactivation

(A) Heatmap showing hierarchically clustered TPM values for differentially expressed isoforms (pvalue $<0.1$ ). Highly significant hits (padj $<0.1$ ) are in bold, while isoforms shaded in purple are also present in Jurkat control group. (B) PSAT1 isoform lacking functionally important exon 8 is differentially downregulated upon HIV reactivation with TNF-alpha. (C) Unproductive PSD4 isoform containing novel intron retention event is predominantly expressed in J-Lat cells prior to HIV induction. Upon HIV reactivation with TNF-alpha, intron retention event is downregulated and productive isoform is upregulated. 
A

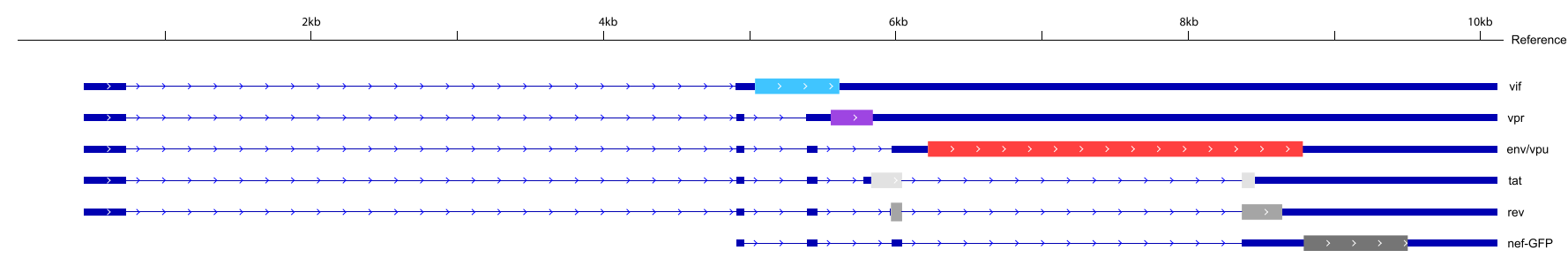

B

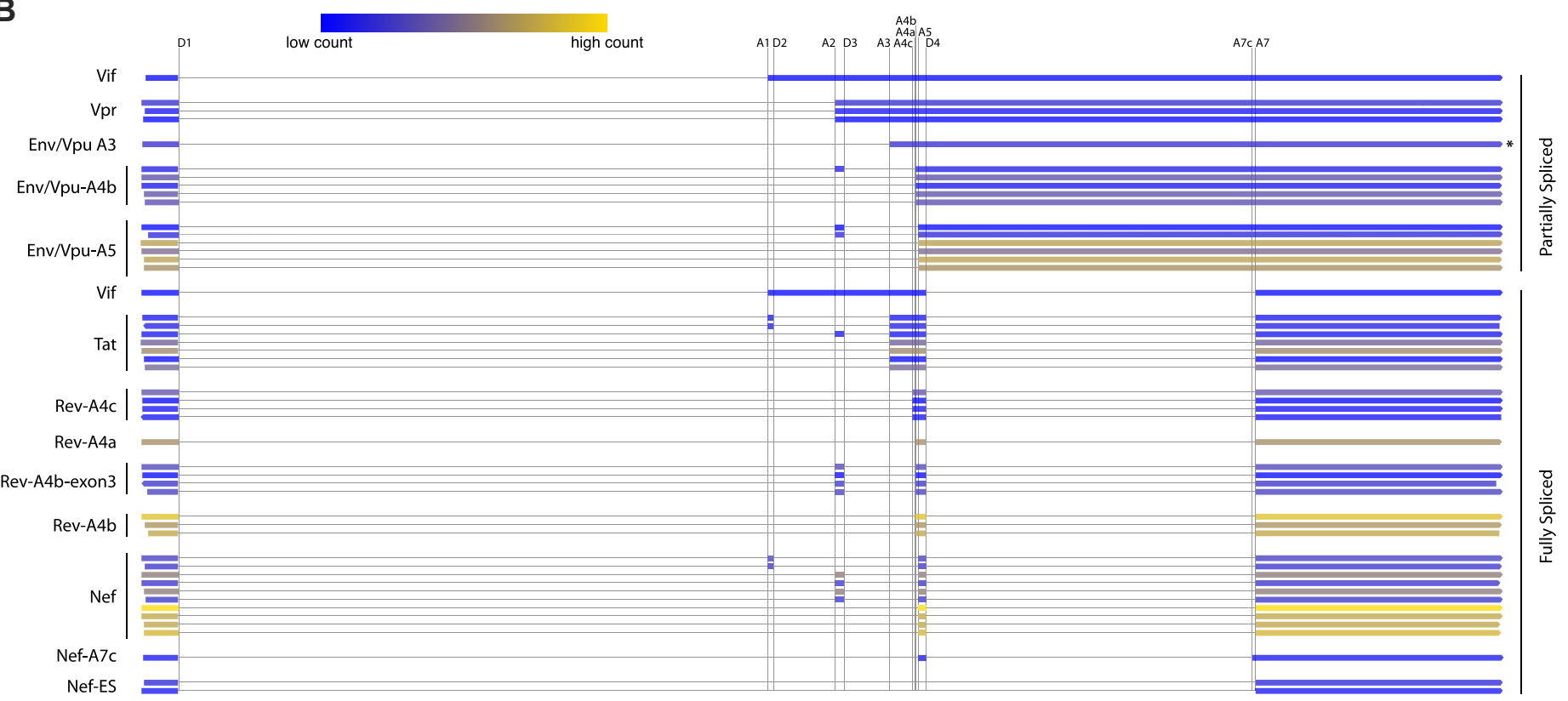

C

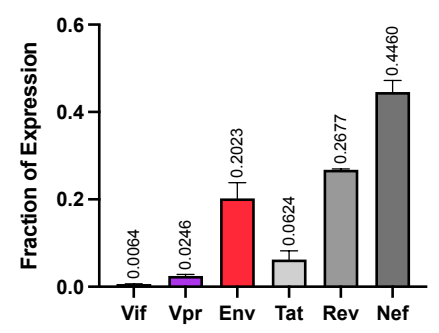

D

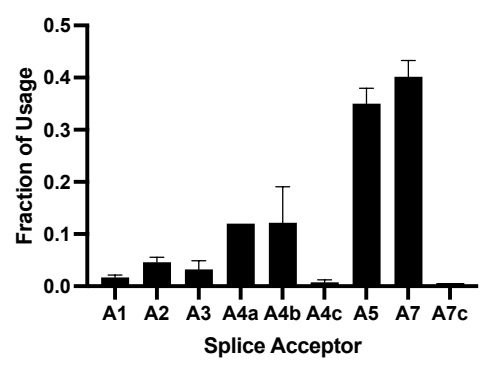

E

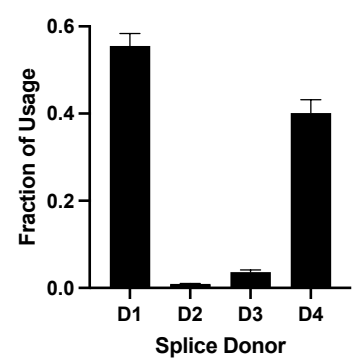

$\mathbf{F}$

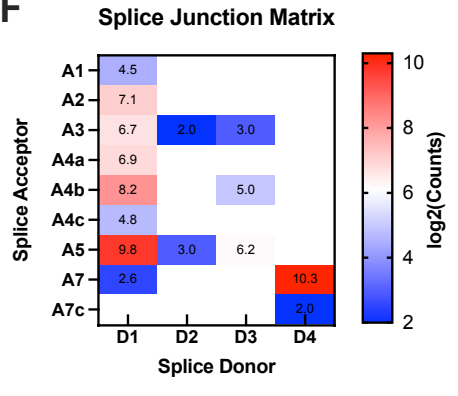

Figure 5 - HIV transcriptional signature, gene expression and splice acceptor/donor usage for TNF-alpha induced viral reactivation in $\mathbf{J}$-Lat 10.6 cells

(A) Idealized splicing structures of HIV genes and their CDS regions. (B) HIV multiexonic isoform clusters observed across four replicates are color coded based on count numbers, isoform clusters are annotated with likely gene expressed and differentiating splice acceptor junction. (C) Gene expression fractions calculated based on counts obtained per isoform cluster, gene assignment based on proximity of ORF to 5' end, and presence of undisrupted CDS. Splice (D) Acceptor and (E) Donor usage. (F) Splice junction matrix with $\log _{2}$ normalized counts shows association and frequency of specific splice donor/acceptor junctions. 


\section{Supplementary Figures and Tables}

A

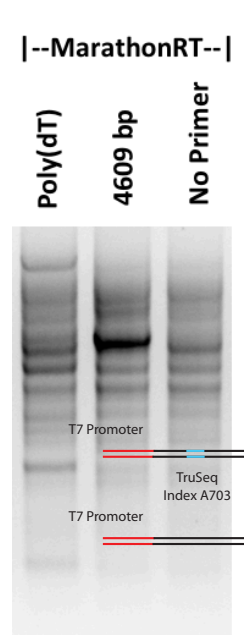

MarathonRT

+ CASPR

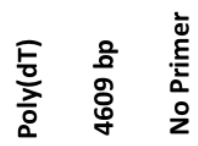

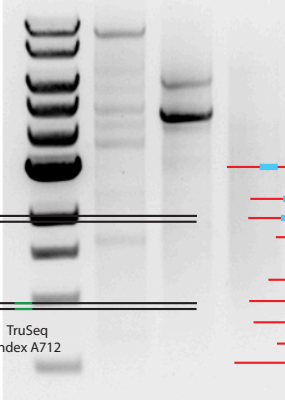

SSIV

SSIV

+ CASPR

퉁 응 훙 융

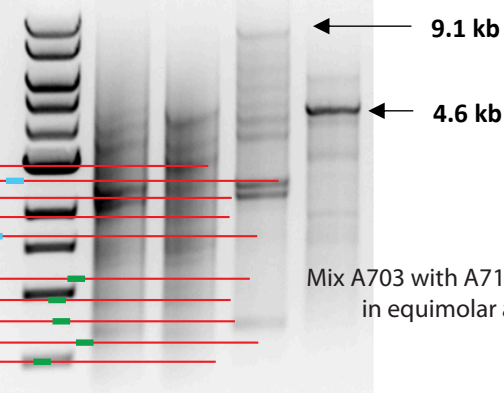

B

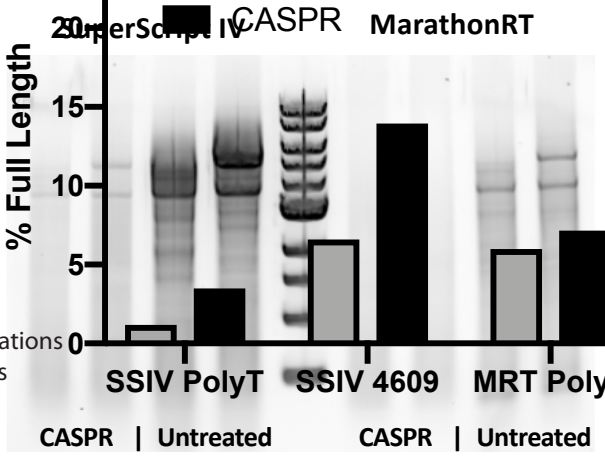

Supplementary Figure 1 - CASPR increases RT specificity of both MRT and SSIV when using a variety of RNA inputs and priming modalities

(A) Agarose gel electrophoresis of SSIV and MRT cDNA products from in vitro transcribed HIV-1 RNA inputs when using Oligo-d(T) or Gene-Specific Priming modalities (B) Agarose gel electrophoresis of SSIV and MRT cDNA products from HEK-293T total RNA when using Oligo-d(T) priming.

A

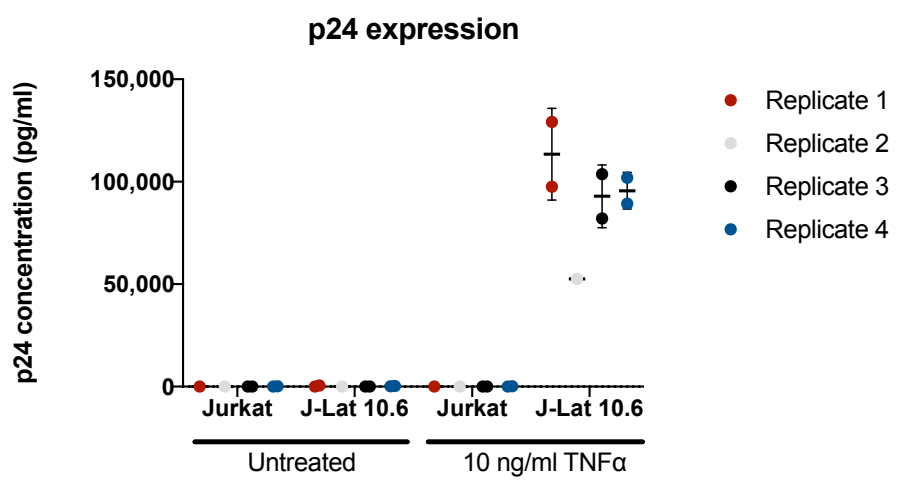

B

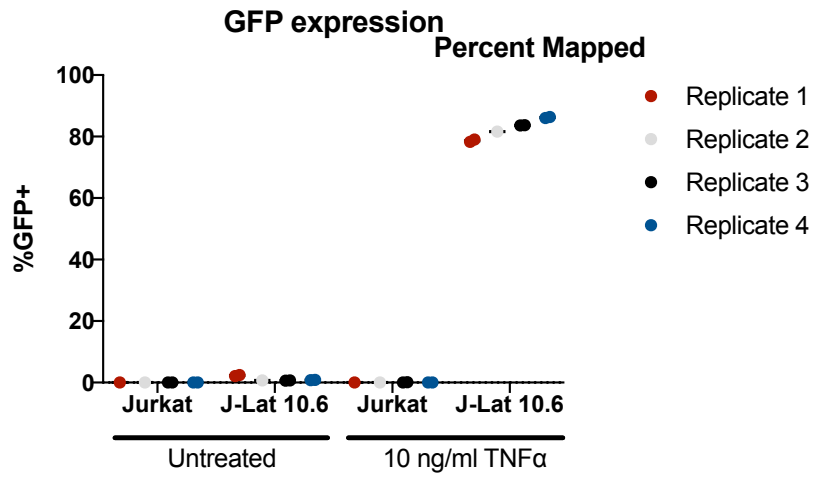

Supplementary Figure 2 - p24 and GFP\% induction in J-Lat 10.6 cells after TNF-alpha induced latency reversal is nominal compared to previous studies

(A) p24 induction from viral supernatants of Jurkat and J-Lat cells activated with TNF-alpha. (B) GFP\% induction for all samples, GFP\% of $75-80 \%$ across J-Lat replicates 

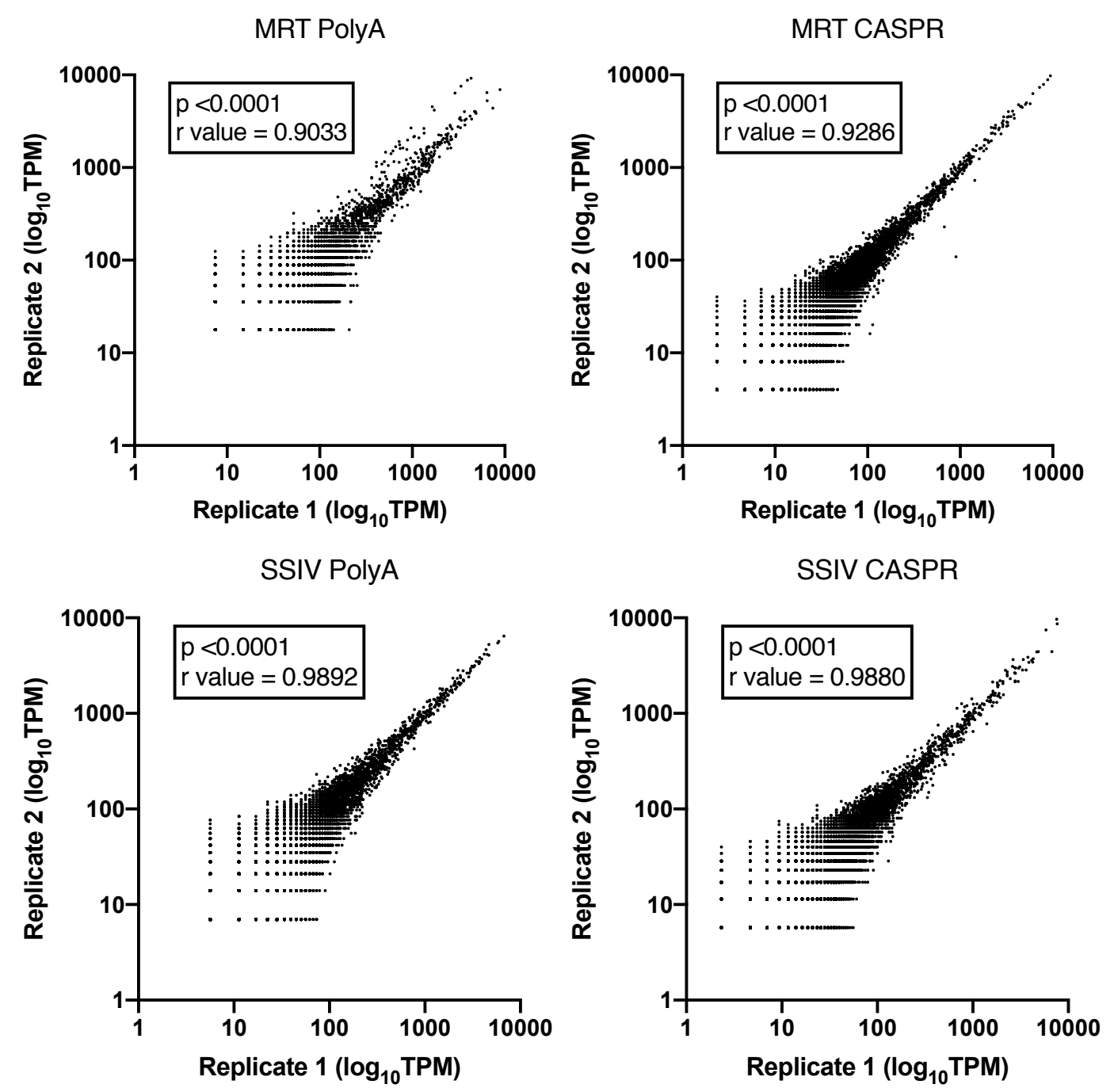

Supplementary Figure 3 - Reproducibility of PolyA and CASPR gene expression TPM values across replicates and treatments

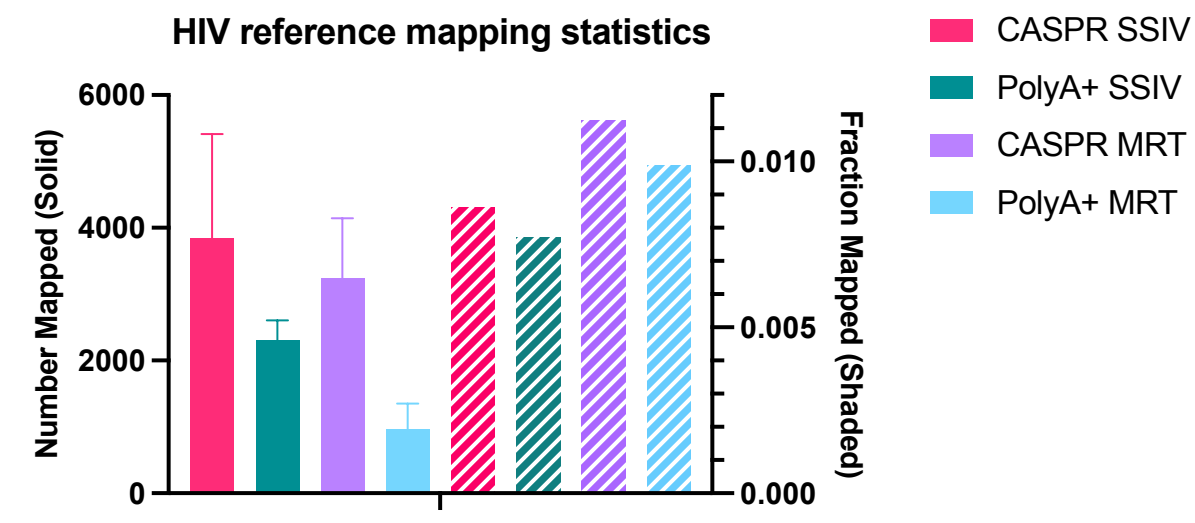

\section{Supplementary Figure 4 - HIV mapping statistics}

Number (solid) and fraction (shaded) of reads mapping to HIV reference sequence 
A

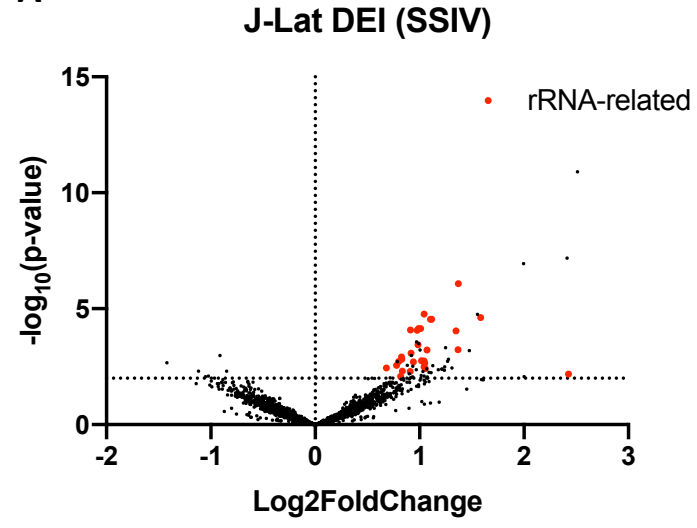

B

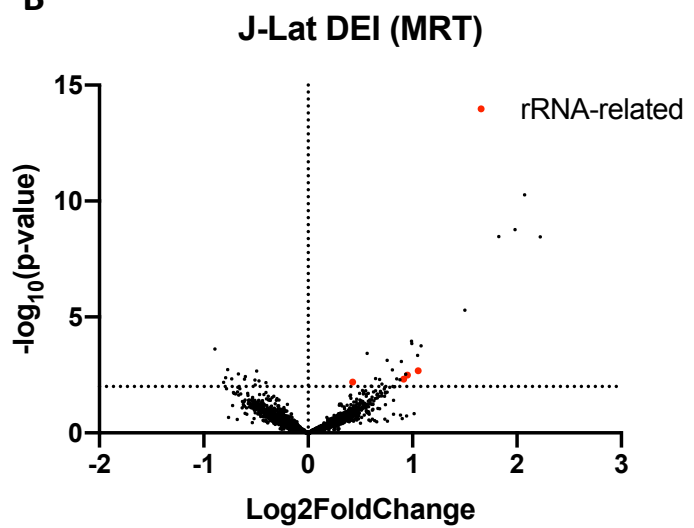

Supplementary Figure 5 - Pilot differential isoform expression (DIE) analysis using CASPR treated RNA inputs, SSIV shows high number of artefactual rRNA related hits compared to MRT

Volcano plots showing high number of rRNA-related DIE hits (red dots) when using (A) SuperScript IV compared to (B) MarathonRT

A

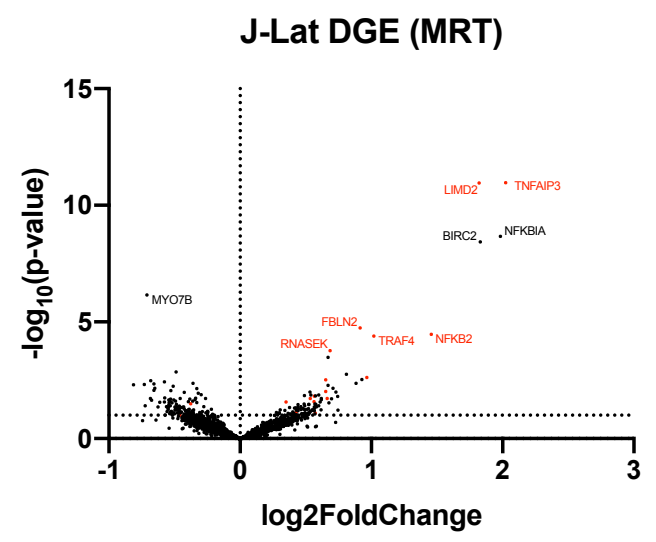

B

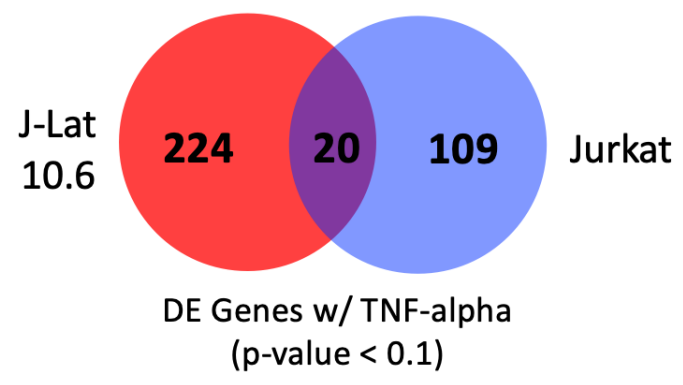

Supplementary Figure 6- Overlap between genes differentially expressed upon TNF-alpha induction in J-Lat case group and Jurkat control Group

(A) Volcano plot showing differentially gene expression (DGE) hits with $p$-value $<0.1$, hits with gene names denote high significance $(\mathrm{p}$-adj $<0.1)$ and those in red denote genes present only in J-Lat case group (B) Venn diagram denoting number of genes showing DGE above threshold for case and control groups, along with degree of overlap. 
bioRxiv preprint doi: https://doi.org/10.1101/2022.01.27.478099; this version posted February 2, 2022. The copyright holder for this preprint (which was not certified by peer review) is the author/funder, who has granted bioRxiv a license to display the preprint in perpetuity. It is made available under aCC-BY-NC-ND 4.0 International license.

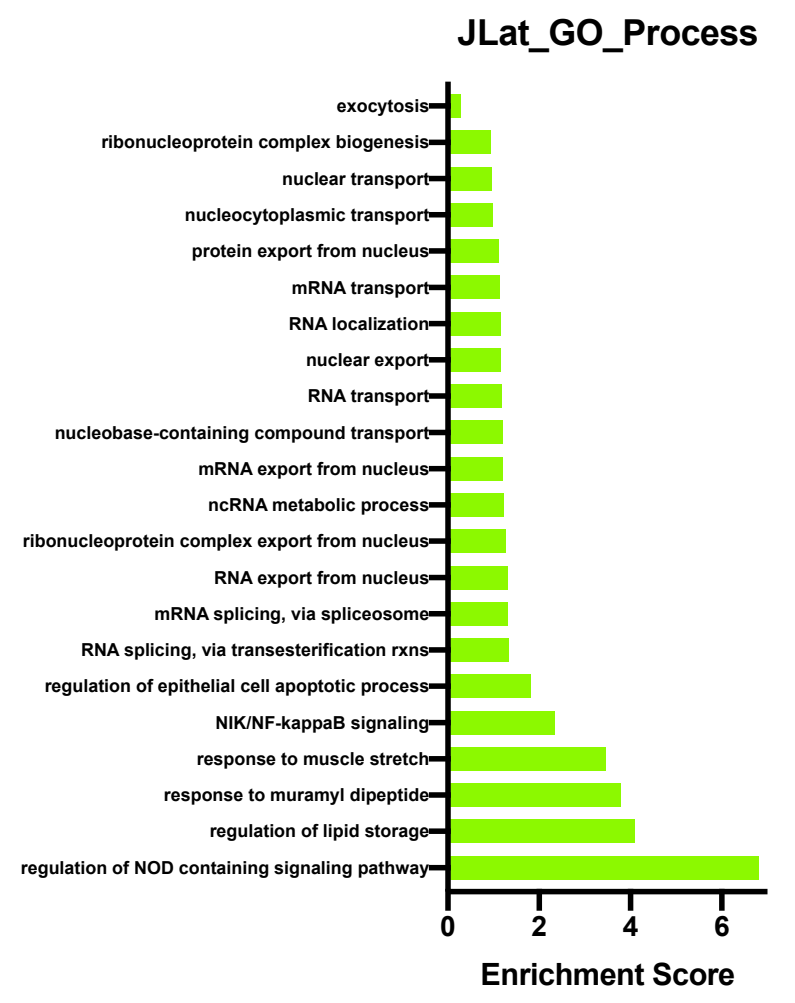

Jurkat_GO_Process

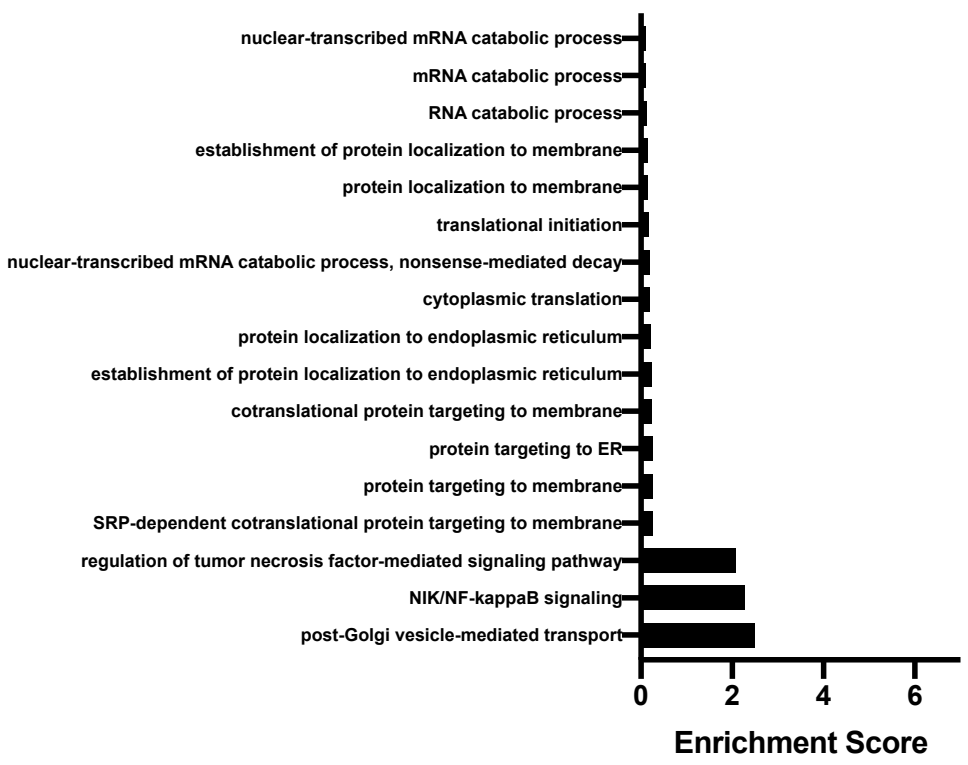

Supplementary Figure 7 - Pathway analysis (GO Biological Process) for Jurkat and J-Lat 10.6 based on $\log _{2}$ Fold Change values TNF-alpha induction 
bioRxiv preprint doi: https://doi.org/10.1101/2022.0127.478099; this version posted February 2. 2022. The copyright holder for this preprint (which was not certified by peer review) is the author/funder, who has granted bioRxiv a license to display the preprint in perpetuity. It is made available under aCC-BY-NC-ND 4.0 International license.

\section{Supplementary Table 1 - HIV Splice Junctions Captured}

\begin{tabular}{|cccc|}
\hline Splice ID & Position (bp) & Type & Notes \\
\hline D1 & 743 & Conserved & \\
D2 & 4962 & Conserved & Non-coding exon 2 \\
D3 & 5463 & Conserved & Non-coding exon 3 \\
D4 & 6045 & Conserved & vpr, tat, rev, nef, vif \\
A1 & 4913 & Conserved & Non-coding exon 2, vif \\
A2 & 5390 & Conserved & Non-coding exon 3, vpr \\
A3 & 5778 & Conserved & tat \\
A4c & 5937 & Conserved & Rev (acceptor C>T) \\
A4a & 5955 & Conserved & Rev (acceptor G>C) \\
A4b & 5961 & Conserved & Rev (major rev) \\
A5 & 5977 & Conserved & Nef, env/vpu (major Env) \\
A7c & 8346 & Novel & Vpr, tat, rev, nef \\
A7 & 8370 & Conserved &
\end{tabular}


bioRxiv preprint doi: https://doi.org/10.1101/2022.01.27.478099; this version posted February 2, 2022. The copyright holder for this preprint (which was not certified by peer review) is the author/funder, who has granted bioRxiv a license to display the preprint in perpetuity. It is made available under aCC-BY-NC-ND 4.0 International license.

\section{Supplementary Table 2 - Read Counts and QC metrics for J-Lat and Jurkat Sequencing Experiments}

\begin{tabular}{|c|c|c|c|c|c|c|c|c|c|}
\hline SAMPLE ID & $\begin{array}{c}\text { RT } \\
\text { CONDITION }\end{array}$ & $\begin{array}{c}\text { TNF } \\
\text { INDUCTION }\end{array}$ & $\begin{array}{l}\text { CELL } \\
\text { LINE }\end{array}$ & READS & BASES & $\begin{array}{c}\text { AVG. } \\
\text { LENGTH }\end{array}$ & $\begin{array}{c}\text { MAX } \\
\text { LENGTH }\end{array}$ & N50 & PAIR ID* \\
\hline 1212-SC-TP-JL & SSIV CASPR & TNFplus & J-Lat & 346,371 & $380,624,842$ & $1,098.90$ & 18,134 & 1,608 & 1 \\
\hline 1212-MC-TP-JL & MRT CASPR & TNFplus & J-Lat & 321,743 & $308,475,236$ & 958.8 & 23,212 & 1,479 & 1 \\
\hline 1212-SC-TM-JL & SSIV CASPR & TNFminus & J-Lat & 413,354 & $470,128,670$ & $1,137.40$ & 21,715 & 1,689 & 2 \\
\hline 1212-MC-TM-JL & MRT CASPR & TNFminus & J-Lat & 342,722 & $348,029,436$ & $1,015.50$ & 20,294 & 1,567 & 2 \\
\hline 1211-SC-TP-JL & SSIV CASPR & TNFplus & J-Lat & 465,339 & $494,378,685$ & $1,062.40$ & 19,100 & 1,583 & 3 \\
\hline 1211-MC-TP-JL & MRT CASPR & TNFplus & J-Lat & 302,825 & $271,089,416$ & 895.2 & 23,629 & 1,400 & 3 \\
\hline 1211-SC-TM-JL & SSIV CASPR & TNFminus & J-Lat & 512,040 & $549,127,704$ & $1,072.40$ & 18,445 & 1,565 & 4 \\
\hline 1211-MC-TM-JL & MRT CASPR & TNFminus & J-Lat & 341,749 & $332,377,217$ & 972.6 & 25,594 & 1,506 & 4 \\
\hline 10232-SC-TP-JL & SSIV CASPR & TNFplus & J-Lat & 207,115 & $273,356,212$ & $1,319.80$ & 20,503 & 1,879 & 5 \\
\hline 10232-MC-TP-JL & MRT CASPR & TNFplus & J-Lat & 297,355 & $289,320,116$ & 973 & 31,113 & 1,344 & 5 \\
\hline 10232-SC-TM-JL & SSIV CASPR & TNFminus & J-Lat & 315,294 & $291,268,332$ & 923.8 & 28,699 & 1,307 & 6 \\
\hline 10232-MC-TM-JL & MRT CASPR & TNFminus & J-Lat & 175,399 & $138,830,006$ & 791.5 & 24,842 & 1,175 & 6 \\
\hline 10231-SC-TP-JL & SSIV CASPR & TNFplus & J-Lat & 510,364 & $752,703,706$ & $1,474.80$ & 27,758 & 2,143 & 7 \\
\hline 10231-MC-TP-JL & MRT CASPR & TNFplus & J-Lat & 516,248 & $536,827,969$ & $1,039.90$ & 27,422 & 1,486 & 7 \\
\hline 10231-SC-TM-JL & SSIV CASPR & TNFminus & J-Lat & 282,062 & $302,421,954$ & $1,072.20$ & 23,163 & 1,596 & 8 \\
\hline 10231-MC-TM-JL & MRT CASPR & TNFminus & J-Lat & 255,840 & $236,887,897$ & 925.9 & 18,757 & 1,403 & 8 \\
\hline 10232A-MC-TP-JL & MRT CASPR & TNFplus & J-Lat & 322,176 & $279,486,945$ & 867.5 & 21,991 & 1,160 & N/A \\
\hline 10232A-MC-TM-JL & MRT CASPR & TNFminus & J-Lat & 560,222 & $489,725,213$ & 874.2 & 29,792 & 1,175 & N/A \\
\hline 210-MC-TP-JU & MRT CASPR & TNFplus & Jurkat & 466,114 & $407,911,711$ & 875.1 & 18,298 & 1,376 & N/A \\
\hline 1211-MC-TP-JU & MRT CASPR & TNFplus & Jurkat & 318,408 & $307,642,349$ & 966.2 & 17,699 & 1,475 & N/A \\
\hline 1023-MC-TP-JU & MRT CASPR & TNFplus & Jurkat & 327,561 & $312,003,518$ & 952.5 & 16,492 & 1,469 & N/A \\
\hline 1023-MC-TM-JU & MRT CASPR & TNFminus & Jurkat & 274,758 & $242,287,201$ & 881.8 & 21,416 & 1,357 & $\mathrm{~N} / \mathrm{A}$ \\
\hline 210-MC-TM-JU & MRT CASPR & TNFminus & Jurkat & 415,924 & $382,228,854$ & 919 & 15,636 & 1,435 & N/A \\
\hline 1211-MC-TM-JU & MRT CASPR & TNFminus & Jurkat & 218,935 & $215,360,547$ & 983.7 & 16,965 & 1,511 & N/A \\
\hline
\end{tabular}

